\title{
„Im Mathematikunterricht muss man auch mit Sprache rechnen!“ - Sprachbezogene Fachleistung und Unterrichtswahrnehmung im Rahmen mathematischer Sprachförderung
}

\author{
Dominik Leiss $(\mathbb{D} \cdot$ Jennifer Plath $(D)$
}

Eingegangen: 20. Januar 2019 / Angenommen: 13. Januar 2020 / Online publiziert: 28. Januar 2020

(C) Der/die Autor(en) 2020

Zusammenfassung In den letzten Jahren ist die Rolle von Sprache für den Erwerb fachlicher Kompetenzen sowie die Relevanz einer durchgängigen Sprachförderung in allen Unterrichtsfächern immer deutlicher geworden. Dies war der Ausgangspunkt dafür, dass im Rahmen des Entwicklungsprogrammes BiSS bundesweit an Schulen Sprachförderung in verschiedenen Unterrichtsfächern implementiert und durch Begleitforschung evaluiert werden sollte. Das Evaluationsprojekt EvaFa untersucht dabei die mathematikspezifische Sprachförderung von Schulen aus dem BiSS-Programm. Entsprechend beschäftigt sich dieser Beitrag mit der Frage, inwiefern Schülerinnen und Schüler, die an einem sprachfördernden Mathematikunterricht teilnehmen, von einem Jahr Sprachförderung profitieren und höhere Textverstehensleistungen bzw. einen größeren Fachwortschatz aufweisen. Hierfür wurden von den Schülerinnen und Schülern eines exemplarischen Sprachförderverbundes bestehend aus acht Klassen in einem Pre-Post-Design u.a. soziodemographische Hintergrundmerkmale, sprachliche Fähigkeiten, die Verfügbarkeit von Fachwörtern, die mathematische Kompetenz, das Textaufgabenverständnis sowie die Einschätzung unterrichtlicher Aspekte längsschnittlich erhoben. Für eine Stichprobe von 133 Schülerinnen und Schüler der 5. und 6. Jahrgangsstufe werden diese Daten näher analysiert, wobei sich zum einen zeigt, dass Schülerinnen und Schüler verschiedene unterrichtliche Aspekte sehr differenziert wahrnehmen und für Veränderungen sensibel sind. Zum anderen zeigen sich beim mathematischen Textaufgabenverständnis signifikante Zuwächse zwischen den beiden Messzeitpunkten im Förderzeitraum.

D. Leiss $(\bowtie) \cdot$ J. Plath

Institut für Mathematik und ihre Didaktik, Leuphana Universität Lüneburg,

Universitätsallee 1, 21335 Lüneburg, Deutschland

E-Mail: leiss@leuphana.de

J. Plath

E-Mail: jenniferplath@outlook.de 
Schlüsselwörter Sprachförderung · Textaufgaben · Modellieren · Evaluation · BiSS

\title{
"In Math Lessons, you also have to Count on Language!"-Language- related Performance and Teaching Perception in the Context of Mathematical Language Support
}

\begin{abstract}
In recent years it has become increasingly clear what meaning language has for the acquisition of professional competences and what relevance a continuous language promotion has in all school subjects. This was the starting point for the BiSS funding program, which implemented language support at various schools in schools across Germany, and which was to be evaluated through accompanying research. One such evaluation project was the EvaFa project. This examined the mathematics-specific language support of schools from the BiSS program. Accordingly, the present article deals with the question of to what extent students who take part in language-promoting mathematics lessons benefit from language support from one year and have higher levels of text comprehension or a larger vocabulary. For this purpose, the longitudinally collected student data from eight classes, which formed a thematically homogeneous language support cluster, were analyzed as part of a pre-post design (including socio-demographic background characteristics, language skills, the availability of technical words, mathematical competence, understanding of word problems and the assessment of teaching aspects). The analysis of the sample (133 pupils in the 5th and 6th grades) shows that pupils perceive different teaching aspects in a very differentiated manner and are quite sensitive to changes in the teaching of mathematics. In addition, the mathematical understanding of word problems shows significant increases between the two measurement times in the funding period.
\end{abstract}

Keywords Language support · Word problems · Mathematical modelling · Evaluation $\cdot$ BiSS

\section{Theoretischer Hintergrund}

Um eine Grundlage für die theoretischen Aspekte der in diesem Artikel beschriebenen Studie zu schaffen, wird im Folgenden zunächst die Rolle von Sprache allgemein für den Fachunterricht (Abschn. 1.1) und daran anschließend den Mathematikunterricht (Abschn. 1.2) mit textbasierten Aufgabenbearbeitungsprozessen als zentrale Sprachhandlungen dargelegt. Hieran anschließend erfolgt die Darlegung des Konzepts eines sprachsensiblen Fachunterrichts (Abschn. 1.3), der sich der Interdependenzen von sprachlichen und fachlichen Leistungen bewusst ist, indem aktuell diskutierte wenn auch nur bedingt empirisch erprobte Prinzipien für einen solchen Unterricht erläutert werden. Abschließend werden für den Bereich des Mathematikunterrichts, in dem erst wenige Studien zu sprachsensiblen Ansätzen bezüglich ihrer Wirksamkeit evaluiert und publiziert worden sind, diesbezügliche Forschungsdesiderata herausgearbeitet (Abschn. 1.4). Aus diesen werden dann die Ziele des 
BISS-Programmes bzw. die Forschungsfragen der in diesem Beitrag vorgestellten Teilstudie des EvaFa-Projekts abgeleitet.

\subsection{Sprache und Fachunterricht}

Seit einigen Jahren erhält die Berücksichtigung von Sprachsensibilität in allen Unterrichtsfächern erhöhte Aufmerksamkeit (vgl. u.a. Schmölzer-Eibinger et al. 2013), sei es in der universitären Aus- und Fortbildung von Lehrkräften oder im Rahmen wissenschaftlicher Forschung (Becker-Mrotzek et al. 2013; Leiss et al. 2017; Koch-Priewe und Krüger-Potratz 2016; Michalak 2014). Dies hängt insbesondere mit den Ergebnissen (inter-)national vergleichender Schulleistungsstudien wie PISA oder DESI zusammen. Hierbei zeigten sich für bestimmte Gruppen deutlich geringere sprachliche Kompetenzen, die wiederum Bildungsbenachteiligung in den Fachleistungen bedingen. Auffallend waren die geringen sprachlichen Leistungen von Schülerinnen und Schülern mit Migrationshintergrund (z. B. Stanat et al. 2010), aber auch die diesbezüglichen Defizite einer relativ großen Gruppe von Schülerinnen und Schülern mit Deutsch als Muttersprache (Naumann et al. 2010). Diese unterschiedlichen Sprachkompetenzen gehen mit Heterogenitätsmerkmalen wie z. B. dem sozioökonomischen Hintergrund (SES) einher, der maßgeblich für die Sprachkompetenz bei Eintritt in die Schule verantwortlich ist und damit einen wichtigen Einflussfaktor für die Bewältigung der sprachlichen Anforderungen in der Schule und den damit verbundenen Bildungserfolg darstellt (Ahrenholz 2017; Walzeburg 2014).

Darüber hinaus weisen zahlreiche andere Forschungsergebnisse darauf hin, dass der Erwerb von fachlichen Kompetenzen und schulischer Erfolg eng mit sprachlichen Fähigkeiten verknüpft sind und Sprachförderung zur Ermöglichung bildungssprachlich gestützten Lernens als Schlüssel zum Erwerb solcher Kompetenzen dienen kann (Becker-Mrotzek et al. 2013). Entsprechend wurde zum Beispiel in einem Erlass Nordrhein-Westfalens, wo der in diesem Artikel analysierte Sprachverbund liegt, die Förderung der deutschen Sprache als Aufgabe des Unterrichts in allen Fächern bezeichnet (Ministerium für Schule und Weiterbildung et al. 1999). In den letzten Jahren sind zwar mittels Konzepten durchgängiger Sprachbildung (z. B. Gogolin und Lange 2011) oder sprachsensiblen Fachunterrichts (z. B. Michalak et al. 2015) erste Ansätze entstanden, um den reflexiven und produktiven Umgang mit Texten, aber auch die Entwicklung und Übung von Lesestrategien im Fachunterricht zu konkretisieren, diese werden jedoch erst in Grundzügen in den Schulen umgesetzt (Becker-Mrotzek et al. 2012). Insbesondere zur Förderung von Kindern und Jugendlichen mit Migrationshintergrund existieren bereits eine Vielzahl an Unterstützungsmaßnahmen in den Schulen (siehe Überblick bei Redder et al. 2010), diese basieren jedoch selten auf theoretisch fundierten und empirisch evaluierten Förderkonzepten (Limbird und Stanat 2006).

\subsection{Sprache und Mathematikunterricht}

Für den Mathematikunterricht liegen vielfältige empirische Befunde dafür vor, dass Fachleistungen signifikant mit der allgemeinen Sprachkompetenz und der Textver- 
stehensleistung zusammenhängen (Duarte et al. 2011; Paetsch et al. 2016; Plath und Leiss 2018; Prediger et al. 2015; Vukovic und Lesaux 2013). Gründe hierfür liegen in den bildungs- und fachsprachlichen Anforderungen des Fachs Mathematik, etwa mit Blick auf das Lesen und Verstehen von Aufgabentexten im Mathematikunterricht (Leiss et al. 2010). Sprachliche Fähigkeiten sind aber auch für den diskursiven Aufbau von Mathematikkompetenz von zentraler Bedeutung. Die Komplexität an zu bewältigenden sprachlichen Anforderungen wird durch die Betrachtung der bivalenten Funktion von Sprache im Mathematikunterricht deutlich: Einerseits dient Sprache in ihrer kommunikativen Funktion als zentrales mündliches und schriftliches Lernmedium und andererseits in ihrer kognitiven Funktion als Werkzeug des Verstehens und Denkens (Maier und Schweiger 1999; Morek und Heller 2012). Zusätzlich ist Sprache im Mathematikunterricht nicht nur Lernmedium, sondern auch Lerngegenstand, da eine Vielzahl an fachspezifischen Wörtern und Redemitteln die Voraussetzung zum Aufbau mathematischen Wissens und mathematischer Fertigkeiten bildet (Pimm 1987).

Längsschnittstudien deuten außerdem auf die kausalen Wirkungen von Sprachkompetenzen hin, wie beispielsweise die Studie von Mücke (2007), die gezeigt hat, dass die allgemeine mündliche Sprachkompetenz von Schülerinnen und Schülern bei Schuleintritt einen signifikanten Einfluss auf die mathematische Kompetenz am Ende der Grundschule hat. Insbesondere für die Bearbeitung von realitätsbezogenen Textaufgaben haben sich sprachliche Kompetenzen als wichtiger Prädiktor für eine korrekte Lösung der Aufgabe erwiesen (Abedi 2006; Heinze et al. 2011; Prediger et al. 2015).

\subsubsection{Sprachkompetenz als potentielle Hürde beim Verstehen mathematischer Textaufgaben}

Die Bearbeitung von realitätsbezogenen Textaufgaben wird als mathematisches Modellieren bezeichnet und ist weltweit als Kompetenz in den Bildungsstandards der Sekundarstufe I zahlreicher Länder verankert (z. B. Leiss und Blum 2006; NCTM 2000; Niss 2003). Im Vergleich zu spracharmen innermathematischen Aufgaben weisen realitätsbezogene Aufgaben in der Regel deutlich geringere Lösungshäufigkeiten und ein größeres Schwierigkeitspotential auf (Abedi und Leon 1999; für einen Überblick siehe Verschaffel et al. 2000), u. a. weil sie neben mathematischen Fähigkeiten insbesondere auch das Verstehen des sprachlichen Aufgabentextes sowie der beschriebenen Aufgabensituation erfordern (Leiss et al. 2010, 2019; Paetsch et al. 2016; Prediger et al. 2015). Ein Modell für die Beschreibung der Bearbeitung solcher realitätsbezogenen Aufgaben stellen Modellierungskreisläufe dar, die zentrale kognitive Elemente des Lösungsprozesses beinhalten. Ein Beispiel für einen solchen ist der siebenschrittige Kreislauf von Blum und Leiss (2005):

1. Bildung eines Situationsmodells durch das Verstehen des Aufgabentextes

2. Bildung eines Realmodells durch das Vereinfachen und Strukturieren der Textinformationen

3. Bildung eines mathematischen Modells durch einen Mathematisierungsprozess

4. Errechnung von mathematischen Resultaten 
5. Bildung von realen Resultaten durch die Interpretation der mathematischen Resultate

6. Validierung der Ergebnisse

7. Darlegung der Ergebnisse

Aus zahlreichen Studien ist bekannt, dass diese Bearbeitungsschritte, auch wenn sie nur bedingt in dieser Reihenfolge in realen Modellierungsprozessen durchlaufen werden, wesentliche kognitive Anforderungen enthalten und entsprechend für Schülerinnen und Schüler potentielle Hürden darstellen können (Galbraith und Stillman 2006). Insbesondere der erste Schritt, das Lesen und Verstehen des Aufgabentextes, bereitet vielen Schülerinnen und Schülern Probleme und kann zu Schwierigkeiten und Fehlern in der restlichen Aufgabenbearbeitung führen (Clarkson 1991; Mayer und Hegarty 1996; Wijaya et al. 2014).

\subsubsection{Sprachkompetenzen, Sprachsozialisation und mathematische Leistungen}

In diesem Kontext wird besonders relevant, dass Publikationen im Rahmen international vergleichender Schulleistungsstudien vermehrt beschrieben haben, dass Schülerinnen und Schüler mit Migrationshintergrund niedrigere Leistungen im mathematischen Bereich erzielen und somit auch geringere Bildungserfolge erreichen als Schülerinnen und Schüler ohne Migrationshintergrund (z. B. Gebhardt et al. 2013; Wendt et al. 2016). Dabei zeigen verschiedene Studien, dass die geringeren Leistungen im Fach Mathematik von Schülerinnen und Schülern durch die Merkmale Nationalität, Migrationshintergrund, Nichtpassung von Familien- und Schulsprache, sozioökonomischer Hintergrund sowie Lesekompetenz erklärt werden können (siehe für einen Literaturüberblick Prediger et al. 2015). Insbesondere bei der Bearbeitung mathematischer Textaufgaben existieren signifikante Differenzen in den Lösungsraten zwischen Schülerinnen und Schülern mit und ohne Migrationshintergrund, welche sich jedoch nicht auf z. B. unterschiedliche kulturelle Erfahrungen zurückführen lassen, sondern vielmehr mit den sprachlichen Fähigkeiten der Schülerinnen und Schüler zusammenhängen (Heinze et al. 2007). Ergänzend hierzu konnte sowohl in Untersuchungen von Prediger et al. (2015) als auch von Plath und Leiss (2018) gezeigt werden, dass für alle Schülerinnen und Schüler neben dem fachlichen Vorwissen die allgemeine Sprachkompetenz unter einer Vielzahl anderer soziodemographischer und sprachlich-biographischer Faktoren den wichtigsten Prädiktor für die korrekte Lösung von Textaufgaben darstellt und die Merkmale sozioökonomischer Hintergrund sowie Migrationshintergrund keine guten Prädiktoren für die Kompetenzen in der deutschen Sprache darstellen. Mit einer Längsschnittuntersuchung arbeiteten Paetsch und Felbrich (2016) neben dem Zusammenhang zwischen Sprach- und mathematischer Modellierungskompetenz weiterhin heraus, dass diejenigen Schülerinnen und Schüler mit niedriger Lesekompetenz durch eine Verbesserung ihrer Lese- und Grammatikfähigkeiten auch ihre Modellierungsleistung steigern konnten. 


\subsection{Sprachsensibler Fachunterricht}

Damit auch den Schülerinnen und Schülern mit geringen sprachlichen Ausgangsvoraussetzungen der Zugang zu bildungssprachlichen Ressourcen und fachliches Lernen ohne sprachliche Schwierigkeiten ermöglicht werden, ist es entsprechend den obigen Ausführungen notwendig, in jedem Fachunterricht Sprachsensibilität zu fokussieren. Bei der Frage, wie diese Sprachsensibilität im Fachunterricht konzeptualisiert werden kann, sind insbesondere zwei Ansätze zentral.

\subsubsection{Das LRT-Framework}

Im US-amerikanischen Raum bietet das Framework for Preparing Linguistically Responsive Teachers (LRT-Framework) von Lucas und Villegas (2011) einen theoretischen Rahmen für die Ausbildung von Lehrkräften mit Blick auf die Förderung im Umgang mit sprachlicher Heterogenität und die Unterstützung von mehrsprachigen Schülerinnen und Schülern. Im Rahmenkonzept werden dabei die Einstellungen der Lehrkräfte (soziolinguistische Bewusstheit, Wertschätzung sprachlicher Vielfalt, Bereitschaft zum Einsatz für Zweitsprachlernende) sowie ihr Wissen und ihre Fähigkeiten (z. B. Wissen bezüglich des Zweitspracherwerbs, Fähigkeit, sprachliche Hürden im Unterricht zu erkennen oder Scaffolding-Maßnahmen) als relevante Faktoren herausgestellt (Lucas und Villegas 2011). Dieses Konzept verbleibt jedoch bisher auf einer normativ-theoretischen Ebene und bedarf erst noch der empirischen Evaluation.

\subsubsection{Prinzipien eines sprachsensiblen Fachunterrichts}

Im deutschsprachigen Raum wird die Forderung nach einer Sprachsensibilisierung in allen Unterrichtsfächern durch Konzepte der durchgängigen Sprachbildung (z. B. Gogolin und Lange 2011) umgesetzt. Diese greifen Aspekte des LRT-Modells auf, sind aber insbesondere dadurch gekennzeichnet, dass eine systematische Auseinandersetzung mit den sprachlichen Anforderungen der fachlichen Lerngegenstände mit den Voraussetzungen, die die Schülerinnen und Schüler zur Bewältigung benötigen und bereits mitbringen, einhergeht. Auch wenn es bisher noch wenige empirisch erprobte Erkenntnisse im Bereich des sprachsensiblen Unterrichts gibt, können zumindest theoretisch plausible Leitlinien formuliert werden, die beschreiben, wie Fachunterricht dazu beitragen kann, sprachliche Kompetenzen der Schülerinnen und Schüler mehr in den Blick zu nehmen und (fach-)spezifisch zu fördern. So schlagen z. B. Schmölzer-Eibinger et al. (2013) sieben Prinzipien vor, die dazu beitragen sollen, Sprache als Medium des Lernens bei der Planung und Umsetzung von Fachunterricht bewusst zu berücksichtigen:

1. Integriertes Sprach- und Fachlernen (Sprachverwendung der Schülerinnen und Schüler beobachten und Unterstützungen anbieten; inhaltliche Verstehens- und Lernprozesse durch gezielte Spracharbeit unterstützen) 
2. Sprachaufmerksamkeit und Sprachbewusstheit (präzises und bewusst gestaltetes sprachliches Handeln, Bewusstmachen und Reflektieren von sprachlichen Phänomenen, Begriffen oder Strukturen)

3. Aktives Sprachhandeln und Interaktion (Anregungen zum aktiven sprachlichen Handeln schaffen; authentische Sprachanlässe schaffen)

4. Transparenz von sprachlichen Anforderungen (neben fachlichen werden auch sprachliche Lernziele expliziert)

5. Systematische sprachliche Unterstützung (zum Beispiel durch die Lehrmethode des Scaffoldings)

6. Fokus auf Schriftsprachlichkeit (Förderung der Produktion längerer, kohärenter mündlicher Äußerungen und schriftlicher Texte)

7. Fokus auf Textarbeit (Vermittlung von Schreib- und Lesestrategien und Förderung der Auseinandersetzung mit längeren Texten)

Diese Prinzipien finden sich in ähnlicher Form in verschiedenen Veröffentlichungen (z.B auch Leisen 2010; Michalak et al. 2015), wurden bisher jedoch empirisch dahingehend nicht überprüft, in welcher Form sich ein solcher sprachsensibler Fachunterricht auf die Wahrnehmung des Fachunterrichts durch Schülerinnen und Schüler oder ihre fachlichen Leistungen auswirkt.

\subsection{Sprachsensibler Mathematikunterricht}

Um das Überwinden sprachlicher Barrieren zu ermöglichen und Schülerinnen und Schülern weitestgehend unabhängig von ihren familiären und sozialen Hintergründen langfristig gleiche Bildungsperspektiven eröffnen zu können, muss auch der Mathematikunterricht derartig sprachsensibel (siehe Abschn. 1.3.2) gestaltet werden und um Konzepte zur fachspezifischen Förderung von Sprach- und Textkompetenz erweitert werden (Leiss et al. 2017). Die Planung von mathematischen Förderprozessen, die sprachliche Facetten (z.B. epistemisches Schreiben, Explizierung fachsprachlicher Spezifika, sprachliche Verständlichkeit, Umgang mit Sprachfehlern etc.) berücksichtigen sowie die (Weiter-)Entwicklung von sprachförderlichen Materialien für den Mathematikunterricht wurde jedoch bisher kaum systematisch dokumentiert und in einen empirischen Zusammenhang mit fachlichen Leistungen von Schülerinnen und Schülern gesetzt. Allerdings lassen sich die bisher bestehenden Ansätze, die als Zielsetzung die Verringerung von sprachlichen Barrieren im Mathematikunterricht (der Sekundarstufe I) verfolgen, grundsätzlich in defensive und offensive Ansätze differenzieren.

\subsubsection{Defensive Ansätze der Sprachförderung im Mathematikunterricht}

Unter defensiven Ansätzen werden diejenigen Ansätze zusammengefasst, die sprachliche Hürden reduzieren oder vermeiden, sodass die Schülerinnen und Schüler Textaufgaben in einer vereinfachten Sprache bearbeiten. Verschiedene Studien deuten zwar an, dass Textaufgaben mit vielen sprachlich komplexen Anforderungen für Schülerinnen und Schülern mit Migrationshintergrund deutlich schwerer zu lösen sind und zu niedrigeren Lösungsraten führen (Abedi et al. 2008; Haag et al. 2013; 
Martiniello 2009; Shaftel et al. 2006; Wolf und Leon 2009), aktuelle Untersuchungen zeigen jedoch auch, dass die sprachliche Vereinfachung von Aufgaben nicht zwingend zu höheren Lösungsraten führen muss bzw. dass die Differenzen zwischen intendiert sprachlich komplexen und sprachlich einfacheren Aufgaben deutlich geringer sind als angenommen (Plath und Leiss 2018; Prediger et al. 2015).

\subsubsection{Offensive Ansätze der Sprachförderung im Mathematikunterricht}

Bei offensiven Ansätzen hingegen steht nicht die Reduktion von sprachlichen Herausforderungen im Mittelunkt. Vielmehr werden diese bewusst zu Lerngegenständen gemacht und Unterstützungsmaßnahmen oder Strategietrainings diesbezüglich entwickelt (Rösch und Paetsch 2011). Während sich defensive Ansätze kurzfristig für schriftliche Testsituationen eignen mögen, fokussieren offensive Ansätze eher den langfristigen Kompetenzerwerb. Bezüglich der in Abschn. 1.3.2 genannten Prinzipien für einen sprachsensiblen Unterricht lassen sich für den Mathematikunterricht Arbeiten im Bereich der Grundlagenforschung (zu Lese- und Schreibprozessen im Fachunterricht sowie zur Spezifik der mathematischen Fachsprache) sowie erste systematische Trainings und Einzelmaßnahmen zur Sprachförderung finden, es fehlen jedoch größere Studien, die zeigen, ob diese Maßnahmen zu einer nachhaltigen Kompetenzsteigerung führen (Schilcher et al. 2017).

Eine Ausnahme stellen hier die beiden Studien von Hagena et al. (2017) sowie von Prediger und Wessel (2018) dar. So untersuchten Hagena et al. (2017) mithilfe eines viermonatigen Lesestrategietrainings, welche Auswirkungen ein fachlich integriertes Strategietraining im Vergleich zu einem separierten Strategietraining, also einem Training, das ausschließlich auf die allgemeine Strategievermittlung fokussiert und nicht in die spezifischen Fachkontexte eingebettet wird, auf die Bearbeitungsleistung bei mathematischen Textaufgaben hat. Hierbei zeigte sich zwar eine Verbesserung der Modellierungskompetenz der Schülerinnen und Schüler in beiden Gruppen, allerdings konnte diese Verbesserung auch bei der Vergleichsgruppe nachgewiesen werden, weshalb Erkenntnisse zu fachbezogenem Strategietraining nach wie vor ein Desiderat darstellen. Prediger und Wessel (2018) vergleichen diskursive und lexikalisch-diskursive Interventionsmaßnahmen für die fachlichen Leistungen von Schülerinnen und Schülern im Bereich Brüche. Hierbei können sie für beide Maßnahmen einen signifikant höheren Lernzuwachs als in der Kontrollgruppe aufzeigen und stellen somit die Relevanz von sprach- und fachintegrierter Förderung heraus. Die Lernzuwächse verlaufen bei einsprachigen und zweisprachigen Lernenden sehr ähnlich, was nahelegt, den Forschungsfokus nicht wie häufig in der Vergangenheit auf zweisprachige Lernende zu legen, sondern Förderungen für alle und insbesondere auch für die einsprachig sprachlich schwachen Schülerinnen und Schüler einzusetzen (Prediger und Wessel 2018).

Zusammenfassend konstatieren die Autorinnen und Autoren, dass noch immer ein großes Forschungsdefizit bezüglich der Effektivität von Maßnahmen und Materialien zur fach- und sprachintegrierten Förderung existiert. 


\section{Hintergrund und Ziele des BiSS bzw. EvaFa-Projektes}

\subsection{Das Sprachförderprojekt BiSS}

Basierend auf dieser theoretisch-empirischen Befundlage setzt das Entwicklungsund Forschungsprogramm Bildung durch Sprache und Schrift (BiSS) an, bei welchem seit 2013 an circa 400 Schulen Maßnahmen zur Diagnostik und Förderung sprachlicher Kompetenzen von Schülerinnen und Schüler in den verschiedenen Unterrichtsfächern begleitet und weiterentwickelt werden (Henschel et al. 2018). Dabei zielt das fünfjährige Forschungs- und Entwicklungsprogramm auf die (Weiter-)Entwicklung von Maßnahmen der Sprachförderung, Sprachdiagnostik und Leseförderung im Elementarbereich, in der Primarstufe und in der Sekundarstufe I sowie auf deren Überprüfung hinsichtlich Effizienz und Wirksamkeit (Henschel et al. 2018). In allen beteiligten Domänen wird die alltags- oder unterrichtsintegrierte Förderung als Schwerpunkt gesetzt. Innerhalb der BiSS-Initiative haben sich zwischen drei und zehn Schulen oder Kindertagesstätten zu regionalen Verbünden zusammengeschlossen, um gemeinsam an Sprachförderkonzepten und -maßnahmen zu arbeiten. Um die Förderkonzepte der sich zu regionalen Verbünden zusammengeschlossenen Schulen zu dokumentieren und bezüglich ihrer Wirksamkeit zu evaluieren sowie den Verbünden die Möglichkeit zur prozessbegleitenden Optimierung zu bieten, wurden ausgewählte Verbünde durch externe Evaluationsprojekte begleitet. Eines dieser Evaluationsprojekte ist das EvaFa-Projekt.

\subsection{Das Evaluationsprojekt EvaFa}

Das interdisziplinäre Forschungsprojekt „Evaluation der Sprachförderung im Fachunterricht der Sekundarstufe I" $(\mathrm{EvaFa})^{1}$ begleitet und evaluiert wissenschaftlich Sprachfördermaßnahmen von sieben Sprachförderverbünden insbesondere für den Mathematikunterricht der Sekundarstufe I aus den Bundesländern Bayern, Berlin und Nordrhein-Westfalen und dokumentiert hierbei fachliche und fachdidaktische Sprachfördermaßnahmen und -materialien, um implizites Handlungswissen der fördernden Akteure explizit zu machen und deren Wirkungen zu analysieren. Die zentrale Gemeinsamkeit der Verbünde besteht darin, dass sie keinen defensiven Ansatz zur Vermeidung von sprachlichen Anforderungen verfolgen, sondern im Sinne eines offensiven Ansatzes einen sprachsensiblen Fachunterricht implementieren wollen. Hierbei werden jeweils verschiedene der unter Abschn. 1.3.2 genannten Prinzipien in den einzelnen Verbünden in den Mittelpunkt der fachlichen Sprachförderung gestellt. Dabei muss jedoch betont werden, dass selbst bei einer Fokussierung auf identische Prinzipien in den einzelnen Verbünden diese sehr unterschiedlich umgesetzt werden und verschiedene Fokussierungen auf die Entwicklung sprachförderli-

\footnotetext{
${ }^{1}$ Das Projekt wird geleitet durch Prof. Dr. Knut Schwippert, Prof. Dr. Astrid Neumann sowie Prof. Dr. Dominik Leiss und gefördert durch das Programm BiSS als gemeinsame Initiative des BMBF, des BMFSFJ, der KMK sowie der JFMK. Damit handelt es sich um ein Teilprojekt der BISS-Evaluationsstrategie, die in 51 ausgewählten Verbünden mit etwa 100 Kindertageseinrichtungen und 200 Schulen systematisch die prozessbezogene Evaluation der Projektziele verfolgt.
} 
cher Materialien, die Vermittlung adäquater Lese- und Schreibstrategien sowie auf eine Professionalisierung der beteiligten Lehrpersonen vorgenommen werden (z.B. Wortschatzarbeit mit Sprachvorbildern oder Wortspeichern, Schaffung von Sprechanlässen, Schreibkonferenzen, Arbeit mit Operatorenlisten).

Hinzukommt, dass die evaluierten Verbünde ihre Sprachbildungsprozesse individuell auf die Bedürfnisse und Schwierigkeiten ihrer Schülerinnen und Schüler abstimmen, sodass Vergleiche z. B. hinsichtlich des Einflusses auf die sprachförderliche Unterrichtswahrnehmung oder Leistungszuwächse zwischen den Verbünden trotz größtmöglicher Flexibilität im formativen Evaluationsprozess nicht zu gewährleisten und auch inhaltlich nur bedingt anzustreben waren. Entsprechend handelt es sich bei dem Evaluationsprojekt - und dies stellt gleichermaßen die größte Stärke und Schwäche der vorliegenden Untersuchung dar - um eine echte Feldstudie einer Vielzahl an Förderkonzepten, die dementsprechend nicht durchgängig den Kriterien für die Durchführung einer standardisierten Intervention unter Laborbedingungen genügen kann. Zudem gilt es zu betonen, dass aufgrund der nur schwer zu kontrollierenden Einflussfaktoren eine entsprechend vorsichtige Interpretation der im Rahmen des Projekts erzielten Ergebnisse geboten ist. Daher fokussiert der vorliegende Artikel auf die Analyse eines prototypischen Verbundes und beschränkt sich entsprechend auf die exemplarische Analyse der Daten eines inhaltlich konsistent arbeitenden Sprachverbundes mit 133 Schülerinnen und Schülern aus acht Klassen des 5. und 6. Jahrgangs. Für die Auswertungen wurden neben mathematischen Leistungen und dem Textaufgabenverständnis auch sprachliche Fähigkeiten, relevante Hintergrundmerkmale sowie die Einschätzungen der Schülerinnen und Schüler in Bezug auf unterrichtliche Aspekte längsschnittlich über den Verlauf eines Sprachförderjahres erhoben. Dies ermöglicht anhand der folgenden Forschungsfragen sowohl eine Analyse der Leistungsveränderung als auch der unterschiedlichen Einschätzung von unterrichtlichen Aspekten durch die Schülerinnen und Schüler im Verlauf einer authentischen Sprachfördermaßnahme.

\section{Forschungsfragen}

Aufgrund der oben beschriebenen empirischen Befundlage untersucht der folgende Beitrag, wie sich exemplarisch in den Klassen eines Sprachförderverbundes die unterrichtliche Wahrnehmung der Schülerinnen und Schüler bezüglich sprachförderlicher Aspekte sowie sprachbasierte Leistungsvariablen im Verlauf der Sprachförderung verändern bzw. welche Einflussfaktoren sich identifizieren lassen. Entsprechend ergeben sich folgende Forschungsfragen, die in drei Bereiche eingeteilt werden können:

Bereich I Veränderung der Unterrichtswahrnehmung der SuS im Rahmen der BiSS-Sprachförderung

Wie verändert sich in der Wahrnehmung der Lernenden nach einem Jahr sprachförderlichem Mathematikunterricht ... 
- ... die sprachliche Verständlichkeit des Mathematikunterrichts?

- ... die generelle Bedeutung, die Lehrpersonen Sprache beimessen?

- ... der produktive Umgang der Lehrpersonen mit Sprachfehlern im Unterricht?

- ... der Einsatz von Textverstehensstrategien bei der Bearbeitung von Textaufgaben?

- ... die schülerbezogene Einstellung gegenüber Sprachhandlungen im Mathematikunterricht?

- ... die schülerbezogene Freude an Textaufgaben?

Bereich II Veränderung der Fachleistungen der SuS im Rahmen der BiSS-Sprachförderung

a) Wie verändert sich die Fähigkeit des Verstehens mathematischer Textaufgaben bei Schülerinnen und Schülern nach einem Jahr sprachförderlichem Mathematikunterricht?

b) Inwiefern können nach einer einjährigen spezifischen Fördermaßnahme in einem Verbund auch verbundspezifische Veränderungen bezüglich sprachbasierter Fachleistungen (z.B. im Bereich des verfügbaren Fach-Wortschatzes; siehe Abschn. 4.2) festgestellt werden?

Bereich III Einflussfaktoren sprachbasierter Leistung(sveränderung)en

Inwiefern hängt die sprachbasierte Fachleistung am Ende der Förderung (FF IIa/b) mit der Bewertung der unterrichtlichen Aspekte 1-5 (FF I) bzw. personenbezogenen Hintergrundvariablen der Schülerinnen und Schüler (wie z. B. Migrationshintergrund, Erstsprache, SES etc.) zusammen?

\section{Methode}

\subsection{Evaluationsdesign}

Dem Evaluationsvorhaben nähert sich das EvaFa-Teilprojekt mit verschiedenen methodischen Zugängen (siehe Abb. 1). Der vorliegende Artikel fokussiert auf die standardisierten Erhebungen zentraler Leistungs- und Fragebogendaten (siehe Abb. 1a). Dabei wurden die zwei Kohorten I und II im Rahmen eines Pre-Post-Testdesgin evaluiert. Zum Messzeitpunkt 1 (MZP 1) befanden sich Kohorte I in der 5. Jahrgangsstufe und Kohorte II in der 6. Jahrgangsstufe. Beide Kohorten hatten zu diesem Zeitpunkt bereits seit über einem Jahr an Sprachfördermaßnahmen im Matheamtikunterricht teilgenommen. ${ }^{2}$ Die Testung zum zweiten Messzeitpunkt (MZP 2) erfolgte nach einem weiteren Jahr Sprachförderung in Jahrgang 6 bzw. 7, wobei ein Großteil der in den unterschiedlichen Kohorten bzw. Jahrgängen eingesetzten Erhebungsinstrumente identisch war bzw. über Ankeritems verfügte (siehe Abschn. 4.3). ${ }^{3}$ In

\footnotetext{
${ }^{2}$ Eine wünschenswerte Messung vor Beginn der Sprachförderung im Verbund ließ sich nicht umsetzen, da das hier beschriebene Evaluationsprojekt später genehmigt wurde als der Sprachverbund.

${ }^{3}$ Im Folgenden wird zur präzisen Beschreibung der verschiedenen Erhebungen die folgende Bezeichnungen verwendet Kohorte-Jahrgang-Messzeitpunkt; also z. B. für die erste Kohorte wird der 2. Messzeitpunkt mit I-6-2 bezeichnet.
} 


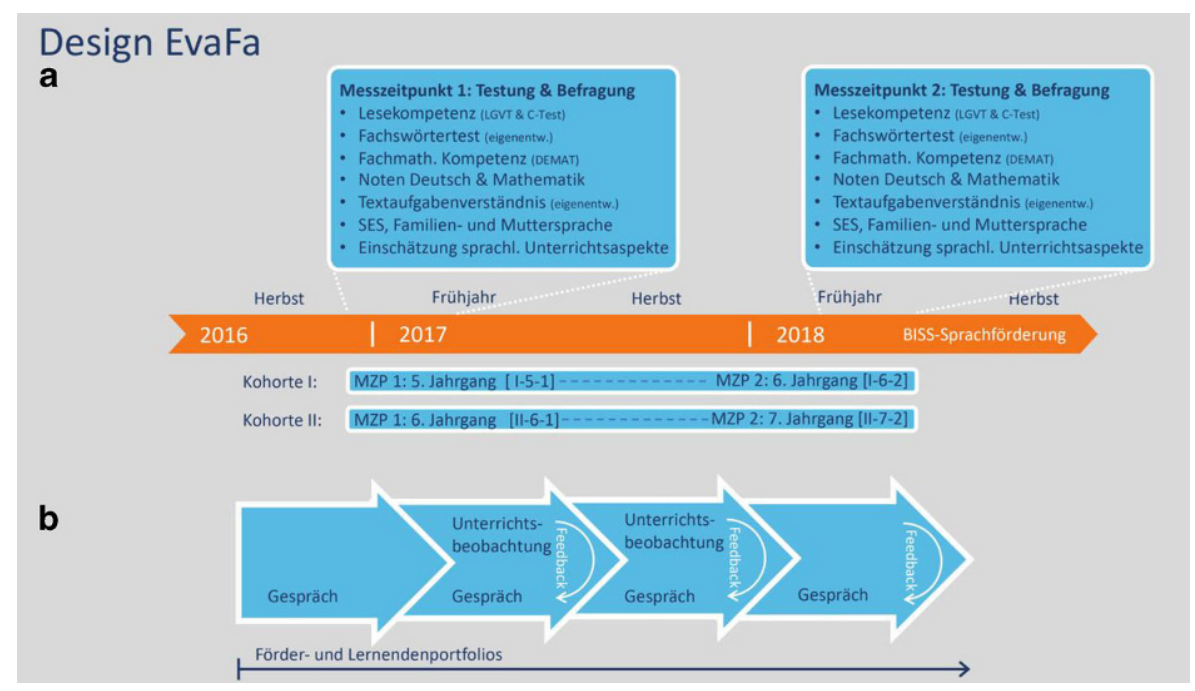

Abb. 1 Design der Evaluation im Rahmen des Projektes EvaFa auf Schulebene

diesem Rahmen wurden die sprachlichen und mathematischen Fähigkeiten der Schülerinnen und Schüler getestet und die Einschätzung verschiedener unterrichtlicher Aspekte sowie relevante Hintergrundmerkmale mit Fragebögen erhoben. Diese wiederholte Testung zum ersten Messzeitpunkt und nach mehr als einem Jahr sprachförderlichem Unterricht zum zweiten Messzeitpunkt ermöglicht eine Untersuchung der Veränderung ausgewählter Aspekte im Verlauf der Förderung.

Ergänzt wurden diese standardisierten Erhebungen (siehe Abb. 1b) durch zwei Schulbesuche mit standardisierten Unterrichtsbeobachtungen, vier leitfadengestützte Gespräche mit den Lehrkräften sowie die organisatorische Unterstützung bei der Verwendung von Förder- bzw. Lernendenportfolios auf Seiten der Lehrpersonen bzw. der Schülerinnen und Schüler. Die Auswertungen dieser Daten werden im Fokus zukünftiger Publikationen stehen.

\subsection{Stichprobe}

Die ursprüngliche Planung des EvaFa-Projekts sah vor mit insgesamt sieben Verbünden bestehend aus 34 Schulen die Evaluation durchzuführen. Da aber für die an BISS teilnehmenden Schulen seitens des BISS-Trägerkonsortiums keine Verpflichtung zur Teilnahme an der Begleitforschung bestand, teilweise eigene Evaluationsmaßnahmen parallel liefen und verschiedene organisatorische Hindernisse auftraten (Schulschließungen, Ausstieg aus BiSS, fehlende zeitlichen Ressourcen, Personalwechsel) konnten nicht in allen Verbünden und Schulen entsprechende Erhebungen durchgeführt werden. Dies führte zu einer selbst für Forschungsprojekte im pädagogischen Feld ungewöhnlich hohen drop-out Rate von $29 \%$ der Schulen zum ersten und $50 \%$ der Schulen zum zweiten Messzeitpunkt.

Entsprechend wird im vorliegenden Beitrag, um einen inhaltlich und statistisch belastbaren Datensatz mit ausreichend Schülerinnen und Schülern, die an einem 
einheitlichen Sprachförderkonzept teilgenommen haben und deren Daten für beide Messzeitpunkten vorliegen, zu erhalten, auf die Auswertung des Verbundes 19_K fokussiert. Dieser Verbund bestand aus insgesamt fünf Gesamtschulen einer Großstadt und fokussierte auf sprachsensiblen Unterricht in acht Klassen der Jahrgänge 5 und 6. Gemeinsam mit der ortsansässigen Universität, welche die Koordination sowie den Großteil der Materialentwicklung übernommen hat, wurden im Rahmen eines iterativen Prozesses mit monatlichen Treffen der Projektbeteiligten Materialien entwickelt, erprobt, überarbeitet und final für Dritte zugänglich gemacht (siehe https://biss-sprachbildung.de).

Für die im Rahmen des Artikels vorgenommene Auswahl dieses Verbundes sprach neben der großen Probandenanzahl insbesondere auch, dass die eigesetzten EvaFaEvaluationsinstrumente sehr gut mit den zwei im Folgenden näher beschriebenen spezifischen Sprachfördermaßnahmen des Verbundes - wie sie von den teilnehmenden Lehrpersonen in den Interviews formuliert wurden - harmonieren; ein Umstand, der aufgrund der hohen Freiheitsgrade in der Gestaltung der Fördermaßnahmen nicht in jedem Verbund realisiert werden konnte:

1. Als ersten Schwerpunkt legt dieser Verbund einen Fokus auf die Wortschatzarbeit und integriert diese auf vielfältige Weise in den Mathematikunterricht. Hierzu gehören z.B. ein Wortspeicher zu den im Unterricht verwendeten Fachwörtern, einheitliche Symbole zu den kommunikativen Anforderungen der einzelnen Aufgaben (z. B. Austausch in Partnerarbeit) sowie Sprachvorbilder in Form von Sprechblasen, die den Schülerinnen und Schülern als Orientierungshilfe für das Bilden von Sätzen oder das Erstellen von Texten dienen können. Um den erarbeiteten Wortschatz auch kontinuierlich im Unterrichtsgeschehen präsent zu halten, werden die Wortspeicher an festen Stellen der Tafel sowie Fachwörterplakate im Klassenraum eingeführt. Die Erklärung mathematischer Fachbegriffe wurde zudem als fester Bestandteil in Klassenarbeiten aufgegriffen.

2. Der zweite Schwerpunkt dieses Verbundes besteht in der Schaffung von möglichst vielen angeleiteten Sprachhandlungen im Mathematikunterricht. Hierfür werden viele mündliche Phasen im Plenum, in Partner- oder in Gruppenarbeit eingeplant. Bei diesen Sprachanlässen geht es nicht nur um die Verwendung des gelernten Fachwortschatzes, sondern auch um die morphologisch-syntaktische Sprachebene. Die Schülerinnen und Schüler werden dazu u.a. angeleitet, mathematische Zusammenhänge mit eigenen Worten zu erklären oder ihre eigenen Lösungswege zu begründen. Hierbei werden als Unterstützungsmaßnahme auch Scaffolds, beispielsweise in Form von vorgegebenen Satzbausteinen, eingesetzt. Zur sprachlich genauen Formulierung von mathematischen Zusammenhängen werden zusätzlich eigene Merksätze von den Schülerinnen und Schüler ins hierfür eingeführte Regelheft geschrieben.

Die umgesetzten Ideen, Konzepte und erstellten Materialien wurden auf gemeinsamen Treffen mit den beteiligten Schulen gegenseitig vorgestellt und besprochen. Zur Dissemination wurde zudem das entwickelte Material auf schulinternen digitalen Plattformen hinterlegt, sodass auch nicht unmittelbar an der Förderung beteiligte Kolleginnen und Kollegen Zugriff auf die Sprachfördermaterialien hatten. 
Tab. 1 Überblick zu zentralen Verbundcharakteristika zu MZP 1

\begin{tabular}{ll}
\hline Verbund 19_K & Daten \\
\hline Schulanzahl & 5 \\
Klassen & $5 \times$ Jahrgang 5 (Klassen-Nr. 1, 4, 6, 7, 8) \& \\
& $3 \times$ Jahrgang 6 (Klassen-Nr. 2, 3, 5) \\
Schüleranzahl & 133 (77 Jahrg. 5 \& 57 Jahrg. 6) \\
Geschlecht (weiblich) & $44,0 \%$ \\
Sozioökonomischer Status & M=2,88 (SD=1,38) \\
& $0-10$ Bücher: 19,6\% (IGLU 5,2\%) \\
& $11-25$ Bücher: 22,3\% (IGLU 9,1\%) \\
& $26-100$ Bücher: 29,5\% (IGLU 26,0\%) \\
& $101-200$ Bücher: 8,0\% (IGLU 14,4\%) \\
& $>200$ Bücher: 20,5\% (IGLU 24,4\%) \\
Immer: 47,2\% & Manchmal: 51,2\% \\
Deutsch als gesprochene Sprache zuhause & $68 \%$ [min. 43\%/max. 83\%] \\
\end{tabular}

Entsprechend setzt sich die Datengrundlage für den vorliegenden Artikel aus fünf zu einem Verbund zusammengeschlossenen Gesamtschulen in Nordrhein-Westfalen zusammen. In den Verbundklassen fanden die Erhebungen des ersten Messzeitpunktes zwischen Dezember 2016 und März 2017 und die Erhebungen des zweiten Messzeitpunktes zwischen April 2018 und Juni 2018 statt, sodass zwischen den Erhebungen etwas mehr als ein Schuljahr lag $(M=69 \text { Wochen, } S D=12)^{4}$. Entsprechend ist für die unten dargestellten Analysen zu bedenken, dass der MZP 2 des 5. Jahrgangs in der zweiten Schuljahreshälfte des 6. Jahrgangs und der MZP 2 des 6. Jahrgangs in der zweiten Schuljahreshälfte des 7. Jahrgangs lag.

Die in Tab. 1 dargestellten Charakteristika des Verbundes zeigen, dass es sich bei der Stichprobe weder um eine spezifische Positiv- noch um eine Negativselektion handelt. Von den 133 Lernenden der Gesamtstichprobe sind 44,0\% weiblich. Als Indikator für den sozioökonomischen Status wurde der fünfstufige Book-at-HomeIndex verwendet (siehe Abschn. 4.3.2). Die gesamte Stichprobe erreicht hier einen Mittelwert von 2,87 (SD=1,34), wobei der Vergleich der Stichprobenverteilung mit der Verteilung bei IGLU zeigt, dass die Verteilung zwar geringfügig nach unten verschoben ist, allerdings lag bei IGLU in Jahrgang 4 noch keine Trennung nach Schulformen vor (vgl. Schulz-Heidorf 2016). Betrachtet man die Lesegeschwindigkeit und das Leseverständnis, so liegen die Schülerinnen und Schüler des Jahrgangs 6 auf einem mittleren Prozentrang (für Jahrgang 5 liegen keinen normierten Daten vor) und auch die Analyse der Muttersprachler sowie der zuhause gesprochenen Sprache zeigt, dass es sich bzgl. dieser Kriterien um durchschnittliche Gesamtschulklassen

\footnotetext{
4 Durch die logistischen Herausforderungen bei über 20 Schulen verteilt auf mehrere Bundesländer Testungen durchzuführen, konnte kein konstanter Zeitabstand zwischen den Messungen gewährleistet werden. Wichtig erscheint in diesem Kontext bzw. in den diesbezüglichen Analysen, dass jeder Proband mindestens ein Jahr Sprachförderung hatte.
} 
handelt (Bodenburg et al. 2018). Entsprechend kann zumindest aufgrund der statistischen Daten davon ausgegangen werden, dass die Bearbeitung der verschiedenen Testinstrumente und Fragebögen durch die Schülerinnen und Schüler nicht durch grundlegende sprachliche Verstehensprobleme gänzlich verhindert wird. Im Einzelfall ist dies nicht auszuschließen, sollte allerdings statistisch nicht relevant werden.

\subsection{Beschreibung der eingesetzten Instrumente}

Die standardisierten Testungen dauerten insgesamt $90 \mathrm{~min}$, wobei in den ersten 30 min das Testheft Sprache (Erhebung der sprachlichen Fähigkeiten und der Schreibkompetenz) bearbeitet wurde, bevor die Schülerinnen und Schüler sich 30 min mit dem Testheft Mathematik (Erhebung der fachmathematischen Kompetenz und der Verstehensfähigkeit beim Lösen von Textaufgaben) beschäftigten (siehe Abschn. 4.3.1). Abgeschlossen wurde die Testung durch den Einsatz eines Schülerfragebogens zur Erfassung von relevanten Hintergrundmerkmalen (siehe Abschn. 4.3.2) sowie von Einschätzungen der Schülerinnen und Schüler $\mathrm{zu}$ ausgewählten sprachsensiblen Aspekten ihres Mathematikunterrichts (siehe Abschn. 4.3.3).

\subsubsection{Erfassung sprachlicher und mathematischer Kompetenzen}

Zur Erfassung von sprachlichen und fachlichen Kompetenzen wurden folgende Instrumente verwendet:

- Sprachliche Fähigkeiten: Zur Erhebung der sprachlichen Fäigkeiten wurde ein C-Test verwendet (vgl. Grotjahn 2014). Beim C-Test werden den Probanden in der Regel fünf Kurztexte mit einer Wortanzahl von 50-70 Wörtern vorgelegt, in denen ab dem zweiten bis zum vorletzten Satz bei jedem dritten Wort systematisch die zweite Worthälfte getilgt wurde und durch die Testpersonen zu ergänzen ist. Da die entstehenden 20-25 Lücken pro Text als weitestgehend unabhängige Testitems skaliert werden können (vgl. Harsch und Hartig 2010), wurde aus testökonomischen Gründen lediglich ein Text mit insgesamt 25 Lücken aus einem erprobten C-Test des Hamburger Instituts für Bildungsmonitoring und Qualitätsentwicklung (IfbQ) verwendet. Die hohe EAP/PV-Testreliabilität von 0,90 scheint dieses Vorgehen zu legitimieren.

- Fachsprache: Im Rahmen eines selbst entwickelten Tests bestehend aus fünf Multiple-Choice-Items wurde in den Jahrgängen 5 und 6 die Bedeutungen der Begriffe Addition, Zehnerstelle, Differenz, Quersumme und Quadrat dergestalt abgetestet, dass die Schülerinnen und Schüler aus zu den Begriffen vorgegebenen Beschreibungen die richtige Charakterisierung auswählen mussten (siehe im Anhang die Items zur Messung des Sprachwortschatzes). Bei diesen Begriffen handelt es sich um solche, die teilweise bereits zu Beginn des 5. Jahrgangs vorliegen müssten bzw. um solche, die in den beiden Klassen 5 und 6 eine starke Rolle im Mathematikcurriculum spielen. Aufgrund des spezifischen EvaFa-Designs erhielten die Schülerinnen und Schüler der 6. Jahrgangsstufe beim MZP 2 eine andere Testversion, so dass hier keine längsschnittliche Messung erfolgen konnte. Auch wenn die Relia- 


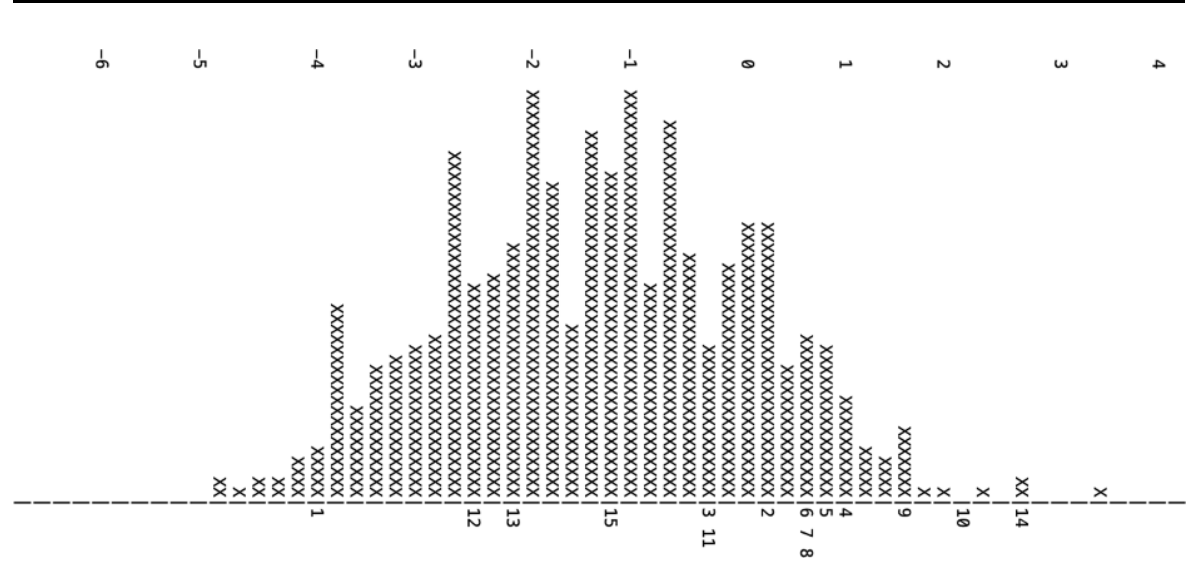

Abb. 2 Verteilung der Probanden des Verbundes 19_K (x-0,2 cases) und der Items (1-15) auf der LogitSkala beim schulischen Kompetenztest DEMAT zum MZP 1

bilität des Tests (EAP/PV Reliabilität 0,44 ) sehr niedrig ist, werden - aufgrund der besonderen Bedeutung für die spezifische Förderung im ausgewählten Verbund sowohl aggregierte als auch auf einzelne Items bezogene Leistungsdaten berichtet.

- Fachmathematische Kompetenz: Zur Messung der mathematischen Kompetenz wurden drei verschiedene Testheftversionen DEMAT $_{A}$, DEMAT $_{B}$ und DEMAT $_{C}$ eingesetzt, die sich aus verschiedenen Items des Deutschen Mathematiktests (DEMAT) 4, 5+ und 6+ zusammensetzten: Der Test DEMAT A $_{\mathrm{A}}$ bestand aus zehn Items

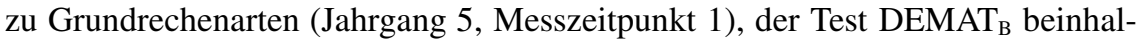
tete vier Items zu Grundrechenarten des Tests $\mathrm{DEMAT}_{\mathrm{A}}$ und fünf weitere Items zum Dreisatz (Jahrgang 5, Messzeitpunkt 2 bzw. Jahrgang 6, Messzeitpunkt 1) und DEMAT $\mathrm{C}_{\mathrm{C}}$ beinhaltete zwei Aufgaben zu Grundrechenarten aus DEMAT $\mathrm{D}_{\mathrm{A}}$, drei Items zum Dreisatz aus $\mathrm{DEMAT}_{\mathrm{B}}$, drei Items zu proportionalen Zusammenhängen und drei Items zu (Dezimal-)Bruchrechnung (Jahrgang 6, Messzeitpunkt 2). Aufgrund der Ankeritems konnten die verschiedenen Testhefte gemeinsam mit dem Programm ConQuest raschskaliert werden. Hierbei wurden die Daten der mathematischen Leistungsstärke (DEMAT) aller Schülerinnen und Schüler im eindimensionalen dichotomen Rasch-Modell skaliert und auf einer Metrik abgetragen, sodass trotz unterschiedlicher Testhefte direkte Vergleiche der geschätzten Personenfähigkeiten (WLE) zwischen den Jahrgängen möglich sind. Der Test zeigt eine EAP/PV Reliabilität von 0,68. In Abb. 2 werden auf der x-Achse die Logit-Werte für die 15 Einzelitems (Zahlen 1-15) abgetragen sowie die Häufigkeit der Schülerinnen und Schüler im jeweiligen Leistungsbereich (x steht für 0,2 Fälle). Dabei wird ersichtlich, dass die Fähigkeitswerte der Schülerinnen und Schüler zumeist im negativen Bereich liegen. Da die mittlere Schwierigkeit der Testaufgaben auf 0 gesetzt wurde (ConQuest-Befehl: constraints=items) wird daran ersichtlich, dass die verwendeten Items geringfügig zu schwer für die getestete Population waren.

- Textaufgabenverständnis: Um die Fähigkeit zu testen, inwiefern die Schülerinnen und Schüler in der Lage sind, eine mathematische Textaufgabe zu verstehen und die relevanten Informationen zu identifizieren, wurden sowohl im Prä- als auch im Posttest vier identische Items eines in dem Projekt „Fach an Sprache an Fach“ (Fa- 
Ein modernes Containerschiff (Preis: 90 Millionen Euro), das $37,5 \mathrm{~m}$ breit und $270 \mathrm{~m}$ lang ist, erreicht eine Höchstgeschwindigkeit von 25 Knoten. Bei einer Geschwindigkeit von 1 Knoten legt ein Schiff pro Stunde 1 Seemeile zurück. Ausgestattet sind die modernen Containerschiffe heutzutage mit riesigen Schiffspropellern. Der bisher größte hergestellte Schiffspropeller hat 5 Flügel und gehört zu einem 85 000-PS-Motor. Insgesamt finden auf einem solchen Containerschiff 7000 Container Platz.

Ina möchte berechnen, welche Strecke (in Seemeilen) das Containerschiff bei Höchstgeschwindigkeit in fünf Stunden zurücklegt.

Unterstreiche all die Zahlenangaben, die Ina wirklich benötigt, um berechnen zu können, wie viele Seemeilen das Containerschiff bei Höchstgeschwindigkeit in fünf Stunden zurücklegt.

Abb. 3 Beispielaufgabe „Containerschiff“ aus dem Projekt „Fach an Sprache an Fach“. (Hagena et al. 2017, S. 12)

$\mathrm{SaF}$ ) entwickelten Instrumentes eingesetzt (Hagena et al. 2017). Jedes Item besteht aus einem Text mit einem Bild (das aber kein Träger wesentlicher Informationen war) sowie einer mathematischen Aufgabenstellung (siehe Abb. 3). Der Text beinhaltet sowohl notwendige als auch überflüssige Informationen zur Beantwortung der Aufgabenstellung. Die Aufgabe der Schülerinnen und Schüler bestand darin, nur diejenigen Zahlenangaben zu unterstreichen, die zur Beantwortung der Aufgabenstellung bzw. zur Durchführung der diesbezüglichen mathematischen Rechnung zwingend erforderlich sind.

Der aus vier Items bestehende Test zum Textaufgabenverstehen wurde unter Verwendung von partial credit Kodierungen im eindimensionalen Rasch-Modell skaliert. Dabei wurde als full credit (2) gewertet, wenn ausschließlich die richtigen Zahlangaben unterstrichen wurden, als partial credit (1), wenn neben allen erforderlichen auch eine überflüssige Angabe unterstrichen wurde und als no credit (0) alle übrigen Bearbeitungen. Dabei zeigte sich, dass die vorgenommene partial credit Kodierung eine gute Modellpassung aufwies, was die Item Characteristic Curve ${ }^{5}$ exemplarisch in Abb. 4 sowie die EAP/PV Reliabilität mit 0,70 verdeutlicht.

\subsubsection{Erfassung von Hintergrundmerkmalen}

Neben Alter und Geschlecht wurden mithilfe eines Schülerfragebogens u. a. folgende Hintergrundmerkmale erhoben:

\footnotetext{
5 Bei einer Item Characteristic Curve werden für die verschiedenen Scores $(0,1,2)$ die empirischen Lösungshäufigkeiten (y-Achse) in Abhängigkeit von der Leistungsstärke der Schülerinnen und Schüler (xAchse) dargestellt. Dabei sind die gepunkteten Linien die empirisch ermittelten und die durchgezogenen Linien die theoretisch erwarteten Werte.
} 


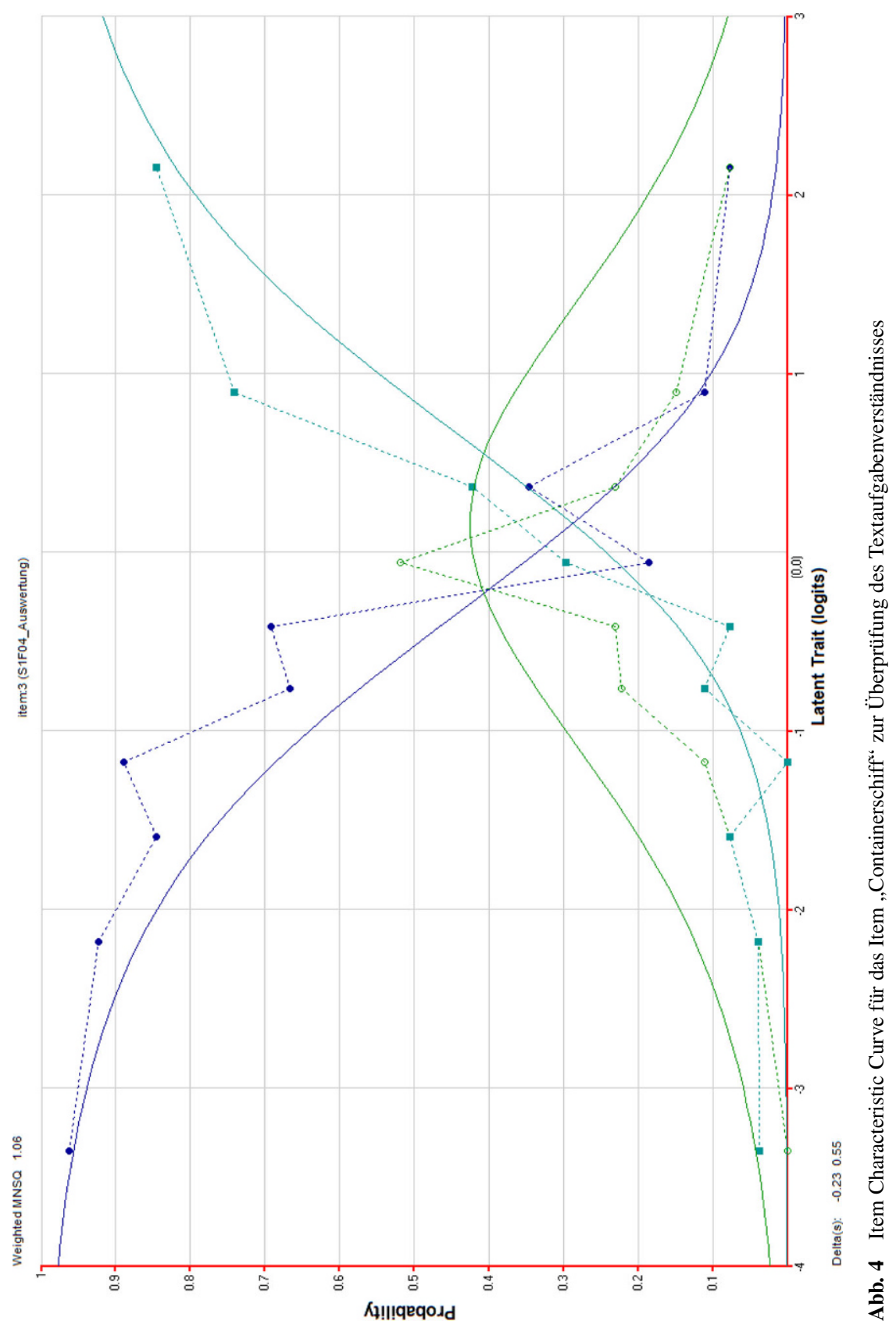


- Sozioökonomischer Status: Als bewährter Indikator für den Sozioökonomischen Status wurde der fünfstufige Book-at-Home-Index verwendet (Bos et al. 2003). Es standen dazu folgende Antwortkategorien zu Auswahl: 1=0-10 Bücher, 2=11-25 Bücher, 3=26-100 Bücher, 4=101-200 Bücher, 5=mehr als 500 Bücher.

- Familiensprache: Die Familiensprache wurde operationalisiert (vgl. Bos et al. 2010) durch die Häufigkeit der Verwendung der deutschen Sprache zuhause (Ausprägungen: Ich spreche immer Deutsch - Ich spreche manchmal Deutsch und manchmal eine andere Sprache - Ich spreche niemals Deutsch).

- Muttersprache: Die Muttersprache wurde adaptiert aus DIPF (2009) dichotom erfasst, indem die Schülerinnen und Schüler angaben, ob sie die deutsche oder eine andere Sprache zuerst gelernt haben.

- Noten: Sowohl für das Fach Mathematik als auch für das Fach Deutsch wurden an jedem Messzeitpunkt jeweils die letzten Zeugnisnoten erhoben.

\subsubsection{Einschätzungen der Schülerinnen und Schüler bezüglich unterrichtlicher Aspekte}

Mithilfe des Schülerfragebogens wurden außerdem die Einschätzungen der Schülerinnen und Schüler zur sprachlichen Verständlichkeit des Unterrichts, zur Bedeutung von Sprache für die Lehrkraft, zum produktiven Umgang der Lehrkraft mit sprachlichen Fehlern, dem Einsatz von Verstehensstrategien sowie die Haltung der Schülerinnen und Schüler gegenüber Textaufgaben erhoben. Diese fünf Aspekte wurden aus den in der Literatur benannten Prinzipien eines sprachsensiblen Fachunterrichts (Abschn. 1.3.2) abgeleitet. Auch wenn eine empirische Überprüfung der Bedeutung dieser Aspekte noch aussteht, ist davon auszugehen, dass sie wesentliche Merkmale eines sprachsensiblen Mathematikunterrichts abdecken. Zur näheren Untersuchung dieser Aspekte wurden folgende fünf Skalen gebildet (siehe im Anhang die Skalen der Schüler*innen-Fragebögen):

- Skala 1: Sprachliche Verständlichkeit des Mathematikunterrichts: Genau wie für die zweite und dritte Skala wurden Items aus der DESI-Studie (Wagner et al. 2009) adaptiert und auf den Mathematikunterricht (statt auf den Deutschunterricht) bezogen. Die Aussagen der Items wurden ebenso wie für die Skalen 2 und 3 von den Schülerinnen und Schülern mithilfe einer 4-Punkt-Likert-Skala ( $1=$ stimmt gar nicht, $2=$ stimmt eher nicht, $3=$ stimmt eher, $4=$ stimmt ganz genau) eingeschätzt.

Die Skala (3 Items, Cronbach's Alpha Testzeitpunkt 1: $\alpha_{\mathrm{t} 1}=0,54$; Cronbach's Alpha Testzeitpunkt 2: $\alpha_{\mathrm{t} 2}=0,70$ ) erfragt, ob die Aufgabenstellungen im Mathematikunterricht klar und verständlich sind, die Lehrkraft anschauliche Beispiele gibt und sich klar und deutlich ausdrückt.

- Skala 2: Allgemeine Bedeutung von Sprache für die Lehrkraft: Die Skala (3 Items, $\left.\alpha_{\mathrm{t} 1}=0,80 ; \alpha_{\mathrm{t} 2}=0,90\right)$ erfragt zum Beispiel, ob die Mathematiklehrkraft auf die Bedeutsamkeit von gutem Deutsch im Alltag oder für das Berufsleben hinweist. 
- Skala 3: Produktiver Umgang der Lehrpersonen mit Sprachfehlern im Unterricht: Die Skala (7 Items, $\alpha_{\mathrm{t} 1}=0,60 ; \alpha_{\mathrm{t} 2}=0,71$ ) enthält beispielsweise Fragen dazu, ob die Lehrkraft sprachliche Fehler als Lerngelegenheiten begreift bzw. die Schülerinnen und Schüler an solchen Stellen produktiv unterstützt.

- Skala 4: Einsatz von Textverstehensstrategien bei der Bildung eines Situationsmodells: Zur Erhebung dieser Skala wurden Items eines Befragungsinstrumentes von Klieme et al. (2005) übernommen. Die Skala (5 Items, $\alpha_{\mathrm{t} 1}=0,72 ; \alpha_{\mathrm{t} 2}=0,71$ ) erfragt über vier Stufen ( $1=$ nie, $2=$ selten, $3=$ oft, 4 = fast immer) u. a. wie häufig bei der Bearbeitung von Textaufgaben gegebene und gesuchte Informationen festgehalten werden oder ein mehrfaches Lesen des Textes erfolgt.

- Skala 5: Einstellungen gegenüber Sprachhandlungen im Mathematikunterricht: Diese Skala $\left(\alpha_{\mathrm{t} 1}=0,62 ; \alpha_{\mathrm{t} 2}=0,72\right)$ wurde aus TIMSS (Bos et al. 2009) adaptiert. Sie erfragt anhand von drei Items und über fünf Stufen $(1=$ stimmt überhaupt nicht, 2=stimmt eher nicht, 3=stimmt teils, teils, 4=stimmt überwiegend, $5=$ stimmt genau) die schülerbezogenen Haltungen bezüglich der aktiven Kommunikation über mathematische Sachverhalte im Unterricht (z.B. „Ich spreche gern mit Mitschülern über meine eigenen Lösungswege.“).

- Weiterhin wurde von den Schülerinnen und Schülern ihre Haltung gegenüber Textaufgaben mithilfe des Items aus TIMSS (Bos et al. 2009) anhand der Aussage „Ich mache gerne Textaufgaben“ und einer Einschätzung über fünf Stufen ( $1=$ stimmt überhaupt nicht, 2 =stimmt eher nicht, $3=$ stimmt teil, teils, $4=$ stimmt überwiegend, $5=$ stimmt genau) erhoben. Analog zum Book-at-Home-Index, der über eine Frage eine grobe, aber empirische erprobte Annäherung an den SES darstellt, dient dieses Einzelitem dazu, die emotionale Haltung mathematischen Textaufgaben gegenüber zu erfassen.

\subsection{Datenauswertung}

Die Dateneingabe sowie die insbesondere auf die Betrachtung von Unterschieden fokussierte Auswertung der Häufigkeiten und Zusammenhänge erfolgten in SPSS (Version 25). Für die Analyse der globalen Lehr-Lernveränderungen durch Sprachförderung (FF I) wurden in Abschn. 5.1 in einem ersten Schritt mithilfe von Faktorenanalysen diejenigen Items der fünf Skalen sprachliche Verständlichkeit, Bedeutung von Sprache, Umgang mit sprachlichen Fehlern, Einsatz von Textverstehensstrategien und Einstellungen gegenüber Sprachhandlungen im Mathematikunterricht identifiziert, die zu möglichst reliablen Skalen führen. Auf dieser Basis wurden anschließend auf Indivudual- und Klassenebene Mittelwerte für die einzelnen Skalen errechnet. Mit diesem Vorgehen können die Einschätzungen zwischen den beiden Messzeitpunkten verglichen werden. Weiterhin wurden für die einzelnen Skalen Streudiagramme erstellt, um die Verteilung der Klassendaten zu den beiden Messzeitpunkten detaillierter darstellen und auch bezüglich ihrer absoluten Werte besser analysieren zu können. Letzteres ist insofern von besonderer Bedeutung, da die Sprachförderung schon zu MZP 1 lief und ggfs. die Einschätzungen der Schülerinnen und Schüler auf einem erhöhten Niveau lagen und nur bedingt mit weiteren Zuwächsen zu rechnen war. 
Tab. 2 Mittelwerte und Standardabweichungen der mathematischen Fachleistungen (DEMAT) der beiden Kohorten am ersten und zweiten Messzeitpunkt

\begin{tabular}{lllll}
\hline Kohorte & Messzeitpunkt & $N$ & Mittelwert (Logit) & SD \\
\hline I-5 & 1 & 76 & $-1,91$ & 1,58 \\
& 2 & 76 & $-1,76$ & 1,33 \\
II-6 & 1 & 57 & $-1,43$ & 1,69 \\
& 2 & 57 & $-0,85$ & 1,72 \\
\hline
\end{tabular}

Für die Untersuchung der Leistungen beim Textaufgabenverständnis (FF IIa) in Abschn. 5.2 wurden t-Tests gerechnet, um mögliche Unterschiede zwischen den beiden Messzeitpunkten auf Signifikanz zu überprüfen. Die Untersuchung verbundspezifischer Veränderungen im Leistungsbereich $(F F I I b)$ fokussierte auf die Verfügbarkeit von Fachwörtern, sodass in diesem Bereich einerseits ein t-Test zu einer möglichen globalen Leistungsveränderng gerechnet und andererseits die Lösungsraten der Einzelitems zu den unterschiedlichen Messzeitpunkten betrachtet wurden. Ergänzend zu den Analysen für die gesamte Stichprobe von 133 Schülerinnen und Schülern wurden analoge Auswertungen auch für die unterschiedlichen Jahrgangsstufen durchgeführt.

Für die Analyse von Einflussfaktoren auf das Textaufgabenverständnis zum Ende der Förderung (FF IIIa) bzw. zur Untersuchung von Fachwortschatz und relevanten Zusammenhangsvariablen ( $F F I I I b$ ) wurden verschiedene Regressionsmodelle gerechnet und in Abschn. 5.3 dargestellt. Hierbei wurden die Leistung im Textaufgabenverstehen am zweiten Messzeitpunkt als abhängige Variable sowie mögliche Einflussfaktoren als Kovariaten betrachtet bzw. aufgrund der ausschließlichen Verfügbarkeit von parallelen Messdaten zum Fachwortschatz am MZP 1 für die Jahrgänge 5 und 6 wurde bzgl. Forschungsfrage $3 \mathrm{~b}$ die Daten des ersten Messzeitpunktes verwendet.

\section{Ergebnisse}

Bevor im Folgenden die in Abschn. 3 angeführten Forschungsfragen beantwortet werden, wird zunächst die in Abschn. 4.2 vorgenommene inhaltlich-formale Stichprobenbeschreibung um die Entwicklung der Fachleistungen im Verlauf der Sprachförderung erweitert. Hierdurch soll eine leistungsbezogene Einschätzung der Probanden parallel zu den Forschungsfragen ermöglicht werden.

Erwartungskonform zeigt sich dabei, dass die mathematischen Fachleistungen der Schülerinnen und Schüler, gemessen durch den DEMAT (siehe Abschn. 4.3.1), sich gemäß der Jahrgangsstufen anordnen lassen (Tab. 2). So geht im Vergleich der Jahrgangsstufen eine spätere Testung innerhalb der Schullaufbahn mit jeweils höheren Leistung im DEMAT einher. Allerdings liegen die Lösungsraten deutlich unter den Normwerten des DEMAT, die jedoch nicht nach Schulformen getrennt vorliegen. Entsprechend kann davon ausgegangen werden, dass es sich im mathematischen Bereich eher um unterdurchschnittlich leistungsstarke Gesamtschülerinnen und Gesamtschüler handelt. 
Als Problem des Messinstrumentes muss jedoch angesehen werden, dass im Verlauf des Sprachförderjahres in Jahrgang 5 scheinbar kein signifikanter fachlicher Leistungszuwachs erfolgt. Eine genauere Betrachtung der vier Ankeritems zeigt, dass die Lösungsraten bei den beiden Aufgaben zur schriftlichen Multiplikation und Division stagnieren, obwohl in diesem Jahrgang curricular viele Wiederholungen im Bereich der Grundrechenarten aus dem Primarstufenbereich und wenige neue Themen behandelt werden. Lediglich bei den beiden Aufgaben zu komplexeren Termumformungen sind auf der Aufgabenebene signifikante Leistungszuwächse feststellbar ( $(150)=-2,653 ; p<0,000$; Effektstärke Cohens d=0,40 bzw. t $(150)=-2,437 ; p<0,000$; Effektstärke Cohens d=0,43).

Erst das deutlicher auf die curricularen Inhalte der Jahrgangsstufe angepasste Testinstrumentarium für den 6. Jahrgang zeigt einen signifikanten Zuwachs der mathematischen Fachleistung von MZP 1 zu MZP 2 (t (113)=-16,445; $p=0,04$; Effektstärke Cohens $d=0,34$ ), allerdings nur bei einer einseitigen Testung, die aber aufgrund der theoretisch gut begründbaren gerichteten Hypothese zulässig erscheint.

\subsection{Veränderung der Unterrichtswahrnehmung der Schülerinnen und Schüler im Verlauf der BISS-Sprachförderung (Forschungsfrage I)}

Um die Frage zu beantworten, inwiefern sich die Wahrnehmung der Schülerinnen und Schüler bezüglich der in Tab. 3 und 4 dargestellten Skalen als relevante Merkmale eines sprachsensiblen Fachunterrichts innerhalb eines Sprachförderjahres verändert, können die Mittelwerte der Skalen für die acht Klassen sowie für die einzelnen Individuen zwischen dem ersten und dem zweiten Messzeitpunkt verglichen werden, wobei jeweils nur diejenigen Schülerinnen und Schüler einbezogen wurden, die an beiden Messzeitpunkten teilgenommen haben. ${ }^{6}$

Hierbei zeigen sich auf Klassenebene für die Skalen der sprachlichen Verständlichkeit des Unterrichts, der Bedeutung von Sprache im Unterricht, dem produktiven Umgang mit sprachlichen Fehlern sowie für die Haltung der Schülerinnen und Schülern gegenüber Textaufgaben keine signifikanten Unterschiede zwischen MZP 1 und MZP 2. Allerdings besteht bezüglich der Haltung gegenüber mathematischen Sprachhandlungen, also einem der zwei Schwerpunkte der Sprachförderung im Verbund 19_K, ein signifikanter Rückgang von 3,40 auf 3,03 (t $(7)=3,661 ; p=0,008)$, was nach Bühner und Ziegler (2017) einem kleinen bis mittleren Effekt von Cohen's $\mathrm{d}=0,37$ entspricht. Die Wertschätzung derartiger Handlungen nimmt demnach bei gleichzeitiger quantitativer Zunahme derartiger Handlungen im Mathematikunterricht im Verlauf der Sprachförderung signifikant ab.

Auch wenn bei den in Tab. 3 dargestellten Analysen die t-Tests aufgrund der sehr starken Datenaggregation mit $N=8$ nur eine sehr geringe Power haben, zeigen die im Folgenden abgebildeten Ergebnisse, bei denen für die maximale Power die Schachtelung der Daten - also die zweite Analyseebene Klasse - bewusst vernachlässigt wurde, dass die Ergebnisse auf Klassenebene bestätigt werden. ${ }^{7}$

\footnotetext{
${ }^{6}$ Entsprechende Berechnungen getrennt für die beiden Kohorten ergaben inhaltlich analoge Ergebnisse.

7 Zur zusätzlichen Absicherung wurde für jede Skala nochmals unter Berücksichtigung der durch die klassenweise Testung entstandenen Datenschachtelung ein lineares Mischmodell (varianzanalytische Analyse
} 


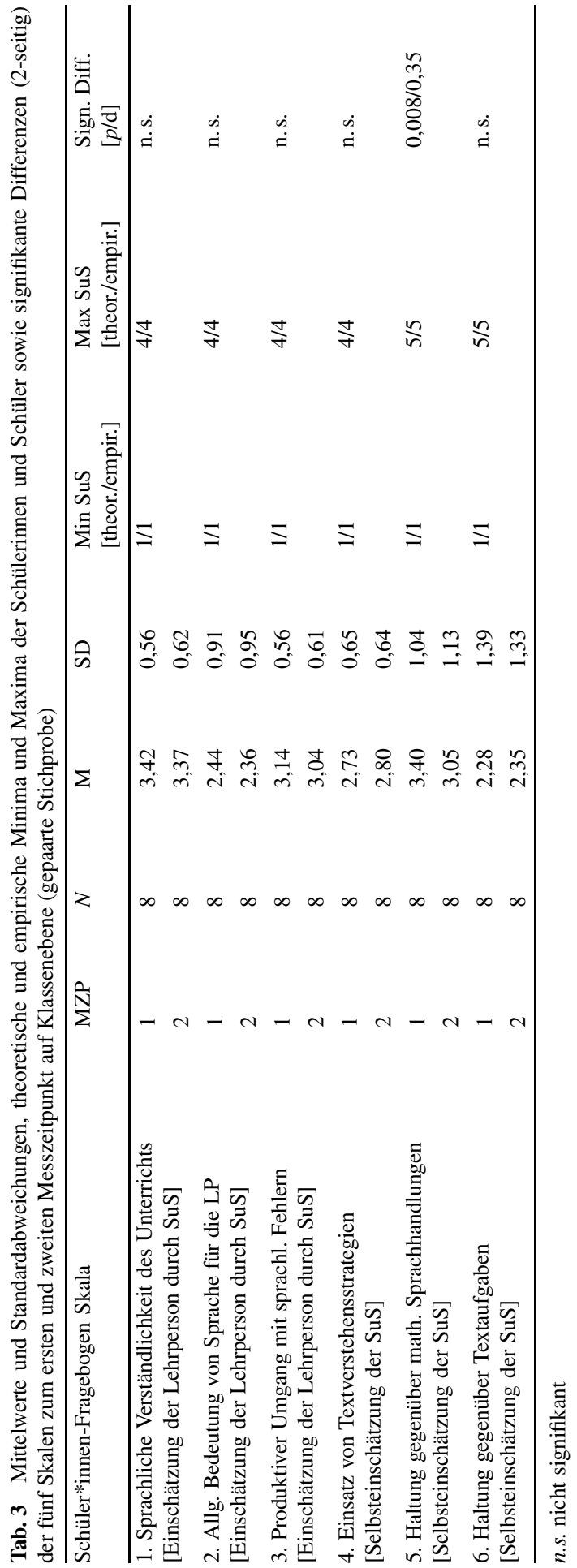


Demgemäß werden zur weiteren Analyse der (qualitativen) Einschätzungen die absoluten Mittelwerte der acht Klassen zu den fünf Skalen sowie zur Frage der Haltung gegenüber Textaufgaben in Abb. 5, 6, 7, 8, 9 und 10 als Streudiagramme abgebildet. Diese Analysen tragen auch dem Umstand Rechnung, dass die untersuchten Klassen zum ersten Messzeitpunkt bereits längere Zeit an Sprachfördermaßnahmen teilgenommen haben und damit die absoluten Werte berücksichtigt werden müssen. Zur besseren Einschätzung der Leistungsveränderung wurde eine Winkelhalbierende sowie zur Orientierung an einer mittleren Antwortkategorie eine Konstante bei 2,5 in die Darstellung eingezogen.

Bei der Einschätzung der sprachlichen Verständlichkeit (Abb. 5) zeigt sich eine grundsätzlich sehr positive Bewertung des Mathematikunterrichts. Bereits beim ersten Messzeitpunkt bewerten alle Klassen die sprachliche Verständlichkeit über dem Skalen-Mittelwert 2,5, wobei die Items der Skala (siehe im Anhang die Items der Skala Sprachliche Verständlichkeit) auf mathematische Aufgabenstellungen und die Ausdrucksweise der Lehrperson fokussieren. Die sprachlich-inhaltliche Verständlichkeit z. B. von Erklärungen zu komplexeren Sachverhalten wird hierbei nicht erfasst.

Die allgemeine Bedeutung, die Lehrkräfte der Sprache Deutsch beimessen, wird durch die Schülerinnen und Schüler tendenziell auf einem mittleren Niveau eingeschätzt (Abb. 6). Auch wenn es in einzelnen Klassen durchaus positive und negative Veränderungen gibt, so fallen diese durchweg moderat aus und liegen unter einer halben Standardabweichung. Hierbei gilt jedoch zu betonen, dass in den Items nicht die spezifische Rolle der Sprache im Mathematikunterricht, also der Bereich der Sprachförderung, sondern eine eher gesellschaftliche Wertigkeit thematisiert wurde.

In Abb. 7 ist zu erkennen, dass die Schülerinnen und Schüler den produktiven Umgang ihrer Lehrpersonen mit sprachlichen Fehlern tendenziell als positiv und lernförderlich einschätzen. Dabei kann die Einschätzung trotz geringfügiger Abweichung bei einzelnen Klassen als stabil angesehen werden. Eine ähnliche Beobachtung lässt sich für den Einsatz von Verstehensstrategien bei der Bearbeitung mathematischer Textaufgaben (siehe Abb. 8) wiederfinden. Obwohl bezüglich des Strategieeinsatzes von den Schülerinnen und Schülern mit einem Mittelwert von 2,74 (MZP 1) bzw. 2,82 (MZP 2) noch Potential nach oben gesehen wird, scheint im Rahmen der hier betrachteten Phase der Sprachförderung keine (signifikante) Steigerung erfolgt zu sein. Betrachtet man die fünf verschiedenen in den Einzelitems getesteten Verstehensstrategien (siehe im Anhang die Items der Skala Einsatz von Textverstehensstrategien), so zeigt sich zumindest für die Strategie gegebene und gesuchte Informationen identifizieren ein signifikanter Zuwachs ( $\mathrm{t}(91)=-2,025 ; p=0,046)$.

Abb. 9 zeigt erneut den oben erwähnten signifikanten Rückgang in der Werthaltung gegenüber sprachlichem Handeln im Mathematikunterricht, macht aber auch deutlich, dass dies auf einem hohen Niveau erfolgt. Darüber hinaus ist auffallend, dass es Klassen gibt, bei denen dieser Rückgang bis zu einer dreiviertel Standardabweichung beträgt und dass für alle drei Bereiche Sprechen, Schreiben und Hören, die jeweils durch ein Item getestet werden, ein signifikanter Rückgang zu verzeichnen

mit Zufallsfaktoren) gerechnet, welche aber die bisherigen Ergebnisse gänzlich bestätigten und entsprechend an dieser Stelle nicht gesondert aufgeführt werden. 


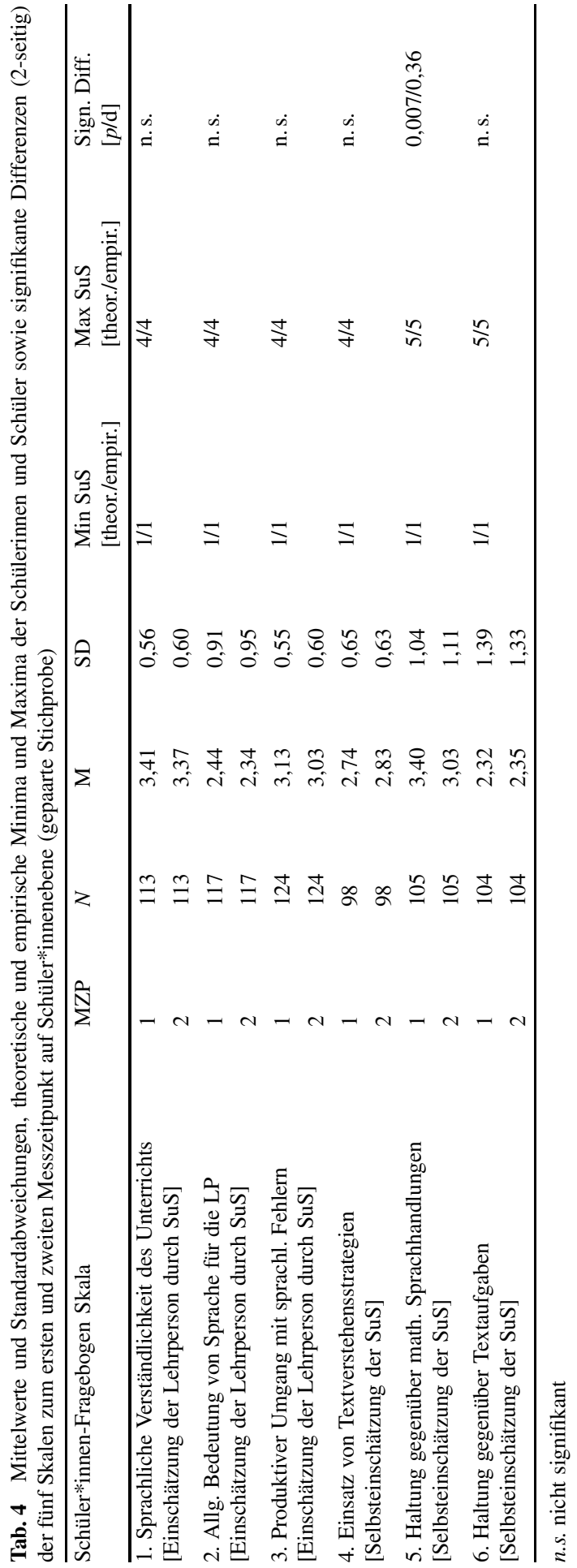




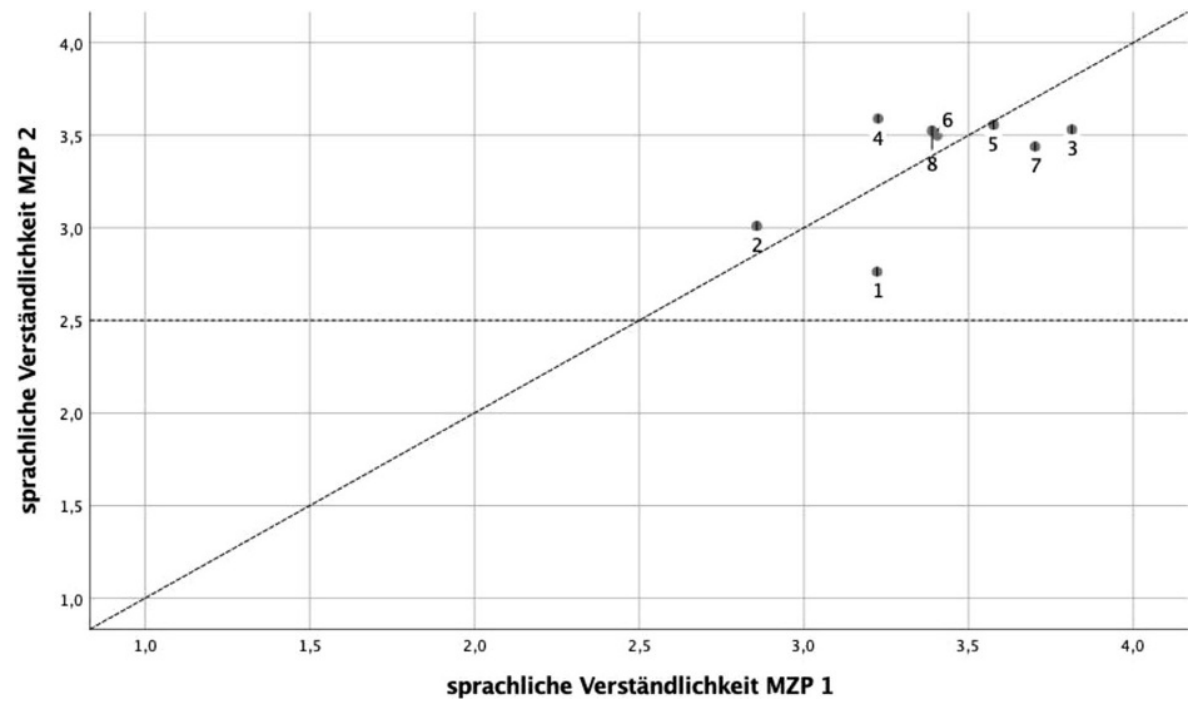

Abb. 5 Streudiagramm: Zusammenhang zwischen der Einschätzung der sprachlichen Verständlichkeit des Mathematikunterrichts im ersten und zweiten Messzeitpunkt

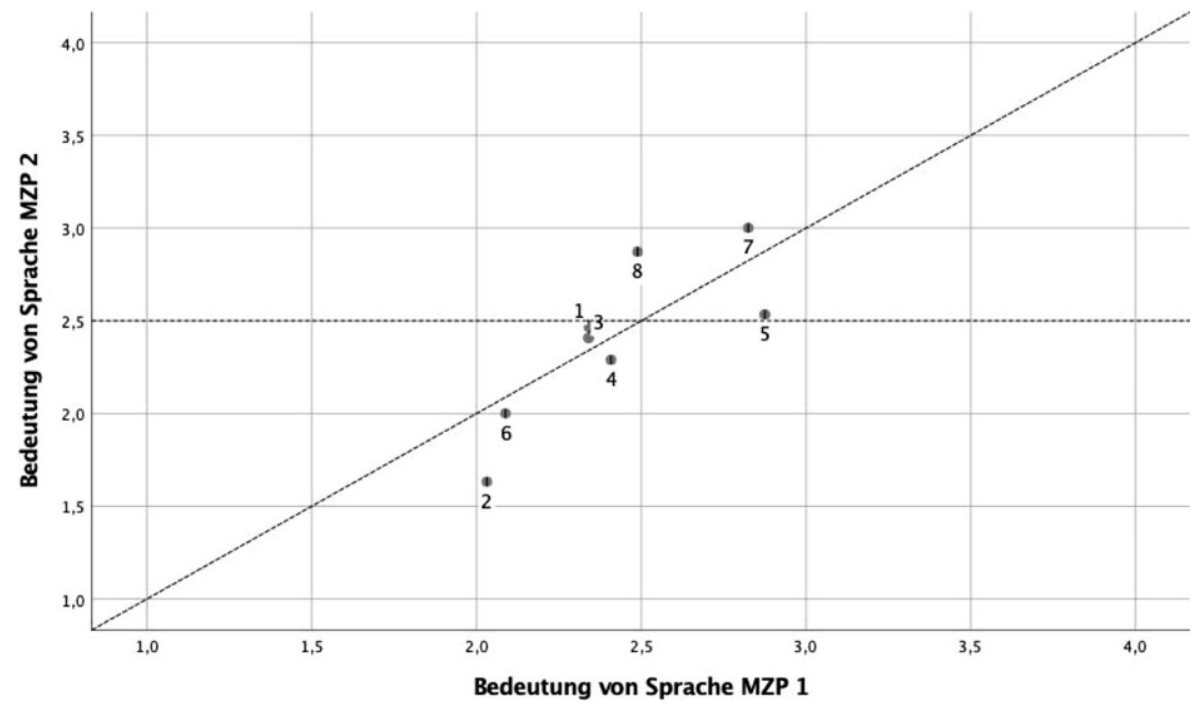

Abb. 6 Streudiagramm: Zusammenhang zwischen der Einschätzung der allgemeinen Bedeutung von Sprache im ersten und zweiten Messzeitpunkt

ist $($ Sprechen t $(100)=2,447 ; p=0,016 /$ Schreiben $\mathrm{t}(104)=2,028 ; p=0,045 /$ Hören $\mathrm{t}(101)=2,070 ; p=0,041)$.

Dass ein Großteil der Schülerinnen und Schüler mathematische Textaufgaben eher ablehnt, lässt sich in Abb. 10 erkennen. Obwohl in allen Klassen im evaluierten Förderzeitraum Strategien und Methoden anhand von Textaufgaben eingesetzt wurden, verändert sich die Einschätzung der Schülerinnen und Schüler derartig text- 


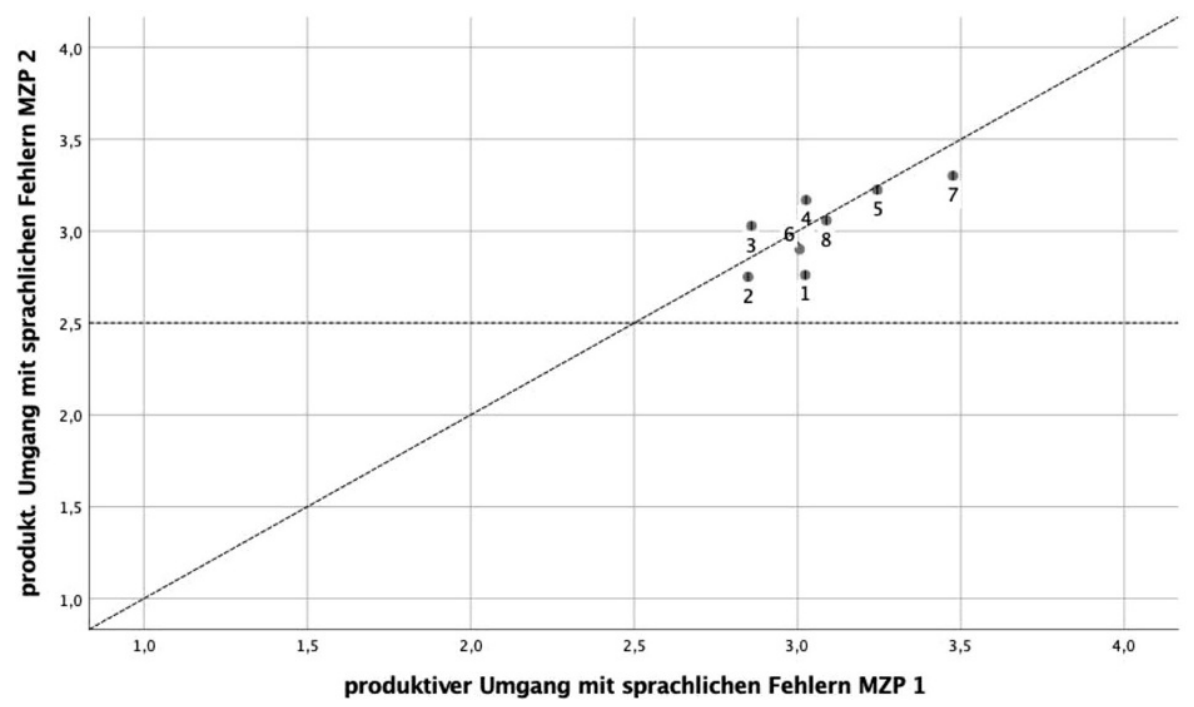

Abb. 7 Streudiagramm: Zusammenhang zwischen der Einschätzung des produktiven Umgangs mit sprachlichen Fehlern im ersten und zweiten Messzeitpunkt

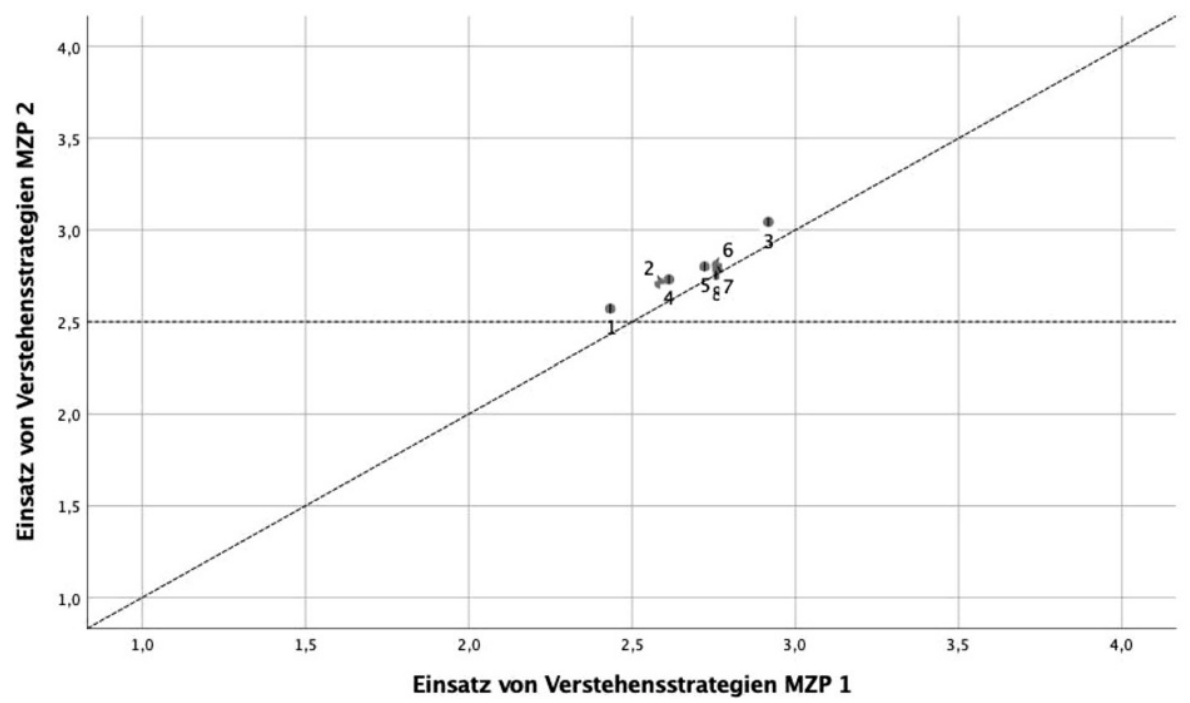

Abb. 8 Streudiagramm: Zusammenhang zwischen der Einschätzung des Einsatzes von Verstehensstrategien bei der Bearbeitung von Textaufgaben im ersten und zweiten Messzeitpunkt

lastigen Aufgaben gegenüber auch nach der Förderung nicht. In den Klassen Nr. 3 und Nr. 7, die im fachlichen (DEMAT) bzw. im sprachlichen Leistungsbereich (CTest) auf Rangplatz 2 und 3 bzw. 2 und 5 stehen, ist sogar ein deutlicher Rückgang - ebenso wie bei der Haltung mathematischen Sprachhandlungen gegenüber - um eine halbe Standardabweichung festzustellen. 


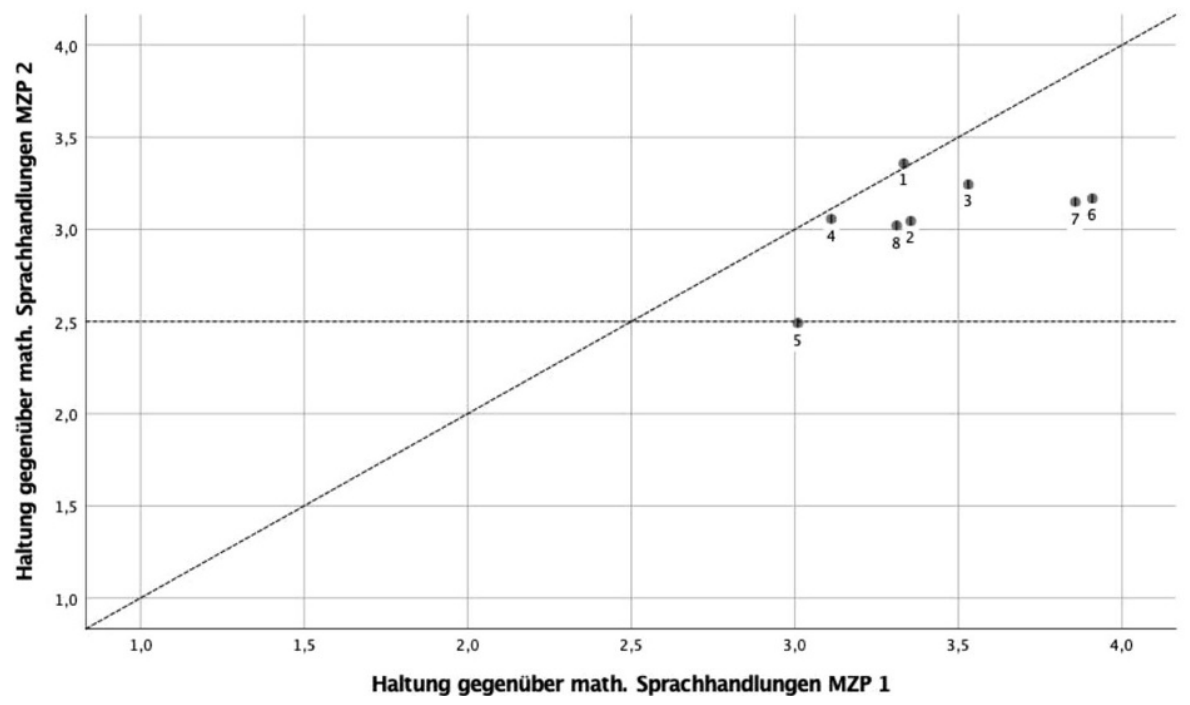

Abb. 9 Streudiagramm: Zusammenhang zwischen der Haltung gegenüber mathematischen Sprachhandlungen im ersten und zweiten Messzeitpunkt

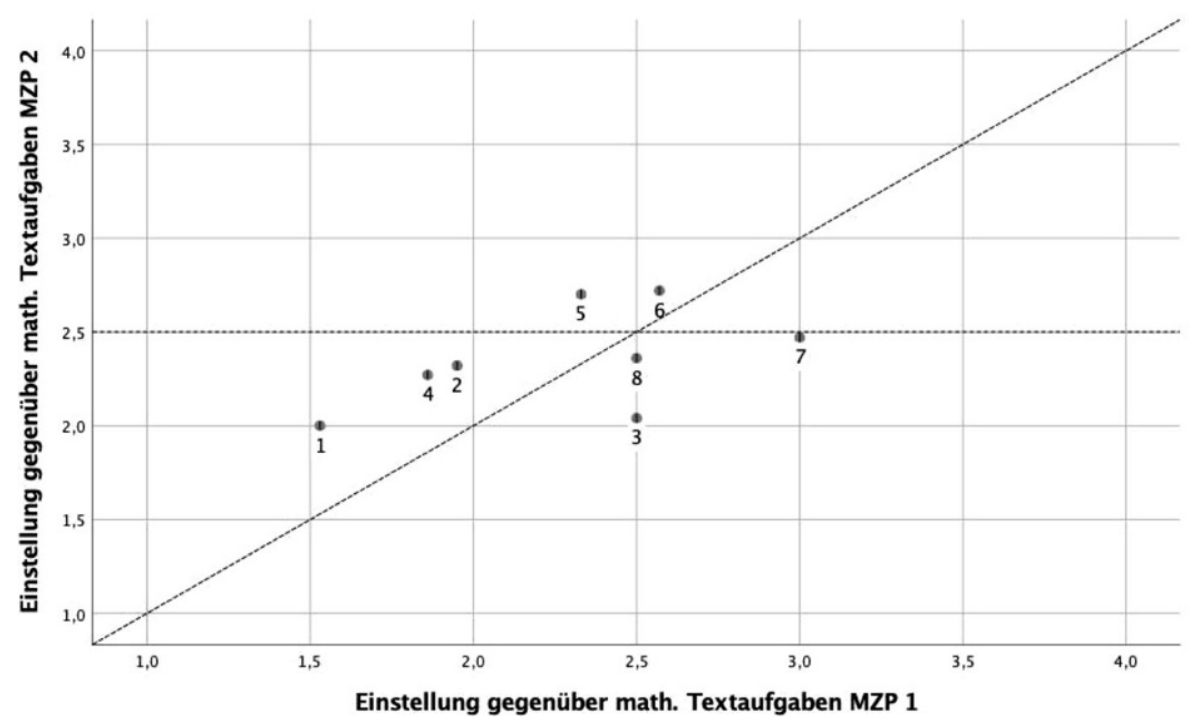

Abb. 10 Streudiagramm: Zusammenhang zwischen der Haltung gegenüber Textaufgaben im ersten und zweiten Messzeitpunkt 


\subsection{Veränderung der sprachbasierten Fachleistungen im Verlauf der BISS- Sprachförderung (Forschungsfrage II)}

\subsubsection{Mathematisches Textaufgabenverständnis}

Die Fähigkeit der Schülerinnen und Schüler, mathematische Textaufgaben zu verstehen (siehe Abschn. 4.3.1), zeigt erwartungskonform einen signifikanten Zuwachs zwischen den beiden Messzeitpunkten ( $\mathrm{t}(132)=-5,10 ; p<0,001)$. Haben die Schülerinnen und Schüler am ersten Messzeitpunkt durchschnittlich einen Leistungswert von $\mathrm{M}=-0,82(\mathrm{SD}=1,32)$, erreichen sie am zweiten Messzeitpunkt einen Mittelwert von $\mathrm{M}=-0,26(\mathrm{SD}=1,50)$. Der t-Test zeigt (siehe Tab. 5), dass diese Differenz signifikant ist und damit die Schülerinnen und Schüler ihre Verstehensleistungen im Verlauf der Förderung verbessern. Dies entspricht einer Effektstärke von Cohen's $\mathrm{d}=0,37$ und damit einem kleinen bis mittleren Effekt.

Zusätzlich wurden diese Differenzen getrennt für die beiden Kohorten analysiert. Dabei zeigt sich zwar, dass erwartungskonform die in Jahrgang 6 beginnende Kohorte II im Vergleich der beiden Messzeitpunkte jeweils signifikant bessere Leistungen erbringt als als die in Jahrgang 5 beginnende Kohorte I (MZP 1: t $(108,01)=-4,44$; $p<0,001 /$ MZP 2: $\mathrm{t}(124,29)=-3,62 ; p<0,001)$. $\mathrm{t}$-Tests konnten dagegen lediglich innerhalb der Förderperiode der ersten Kohorte I statistisch signifikante Fortschritte in der Fähigkeit, eine Textaufgabe zu verstehen, mit einem kleinen bis mittleren Effekt belegen (siehe Tab. 6).

Betrachtet man die Entwicklung der Fähigkeit, mathematische Textaufgaben zu verstehen bzw. sich die für die Beantwortung der Fragestellung benötigten Informationen aus dem Text zu entnehmen auf Klassenebene (siehe für die Klassennummern - Tab. 1), so sind insbesondere drei Aspekte von besonderem Interesse (siehe Abb. 11).

- Die Leistungsstreuung der Klassenmittelwerte innerhalb der beiden Jahrgänge ist jeweils größer als eine Standardabweichung.

- Diese Streuung ist so groß, dass Klasse Nr. 2 der Kohorte II bezüglich der Kompetenz, Textaufgaben zu verstehen eher in Kohorte I zu verorten wäre.

- Die mit Abstand leistungsschwächste Klasse im Verbund erzielt innerhalb eines Jahres Sprachförderung keine messbaren Fortschritte bezüglich ihrer Kompetenz Textaufgaben zu verstehen.

\subsubsection{Mathematischer Fachwortschatz}

Insgesamt zeigt sich, dass bei den Schülerinnen und Schülern der Kohorte ${ }^{8}$ innerhalb der analysierten Förderperiode ein signifikanter Zuwachs ihres Fachwortschatzes $\mathrm{zu}$ verzeichnen ist (t $(75)=-2,822 ; P=0,006)$. Dieser Zuwachs entspricht mit Cohen's $d=0,32$ einem kleinen bis mittleren Effekt. Analysiert man das für

\footnotetext{
${ }^{8}$ Für die 7. Jahrgangsstufe - also dem zweiten Messzeitpunkt der Kohorte II - wurden im Rahmen des Projektdesigns für alle Sprachverbünde andere Fachwortschatztests verwendet, so dass hier keine inhaltlich interpretierbare Zuwächse untersucht werden können.
} 
Tab. 5 t-Test für gepaarte Stichproben (2-seitig) auf Differenzen der Verstehensfähigkeiten der Schülerinnen und Schüler bei Textaufgaben zwischen MZP 1 und 2

\begin{tabular}{llllllll}
\hline & $N$ & M & SD & SE & T & Df & Sig \\
\hline $\begin{array}{l}\text { Differenz } \\
\text { Verstehensfähigkeiten }\end{array}$ & 133 & $-0,56$ & 1,27 & 0,11 & $-5,10$ & 132 & 0,000 \\
MZP 1 \& 2 & & & & & & & \\
\hline
\end{tabular}

Tab. 6 Mittelwerte, Standardabweichungen, Signifikanzniveau (2-seitig) und Effektstärke für die Verstehensfähigkeiten im ersten und zweiten Messzeitpunkt, vergleichend für die Kohorten I und II

\begin{tabular}{lllllll}
\hline & MZP & $N$ & M & SD & $p$ & Cohen's d \\
\hline Verstehensfähigkeit & 1 & 76 & $-1,24$ & 1,13 & $<0,001$ & 0,41 \\
Kohorte I & 2 & 76 & $-0,64$ & 1,47 & & \\
Verstehensfähigkeit & 1 & 57 & $-0,26$ & 1,35 & n.s. & 0,37 \\
Kohorte II & 2 & 57 & 0,26 & 1,39 & & \\
\hline
\end{tabular}

n.s. nicht signifikant

Tab. 7 Effekte von unterrichtlichen Aspekten und Personenmerkmalen auf das Textaufgabenverständnis am zweiten Messzeitpunkt

\begin{tabular}{|c|c|c|c|c|}
\hline $\begin{array}{l}\text { Abhängige Variable: } \\
\text { Textaufgabenverständnis (2. MZP) }\end{array}$ & $\begin{array}{l}\text { Regressionskoeffizient } \\
\beta\end{array}$ & Standard. $\beta$ & SE & Sig \\
\hline (Konstante) & 0,121 & - & 0,134 & - \\
\hline Textaufgabenverständnis (MZP 1) & 0,481 & $0,424 * * *$ & 0,102 & 0,000 \\
\hline Sprachl. Fähigkeit - C-Test (MZP 1) & 0,282 & $0,268 * *$ & 0,095 & 0,003 \\
\hline
\end{tabular}

$N=133, \mathrm{R}^{2}=0,40$

*** Signifikanzniveau 0,001, ** Signifikanzniveau 0,01, * Signifikanzniveau 0,05, n.s. nicht signifikant

die Untersuchungszeit geltende Kerncurriculum des Bundeslandes (Ministerium für Schule, Jugend und Kinder des Landes Nordrhein-Westfalen 2004), so wird dort explizit (Addition, S. 20 \& Quadrat, S. 18) bzw. aufgrund der Inhaltsbeschreibungen implizit (Zehnerstelle, Differenz und Quersumme, S. 20) erwähnt, dass die Schülerinnen und Schüler alle fünf getesteten Begriffe bis zum Ende der Jahrgangsstufe 6 sachangemessen verwenden können müssen. Die Abb. 12 verdeutlicht einerseits den signifikanten Leistungszuwachs, aber andererseits auch, dass in Jahrgang 6 sowohl die Schülerinnen und Schüler der zweiten Kohorte zum MZP 1 (II-6-1), der in der ersten Hälfte des 6. Jahrgangs lag, als auch die Schülerinnen und Schüler der ersten Kohorte zum MZP 2 (I-5-2), der in der zweiten Hälfte des 6. Jahrgangs lag, dieses Lernziel nur bedingt erfüllen. Je nach Fachwort können zwischen 28,9 und 75\% derjenigen Schülerinnen und Schüler, die in der 5. und 6. Jahrgangsstufe eine Förderung, die explizit auf die Vermittlung von Fachwörtern abzielt, erhalten haben, keine adäquaten Beschreibungen für die Fachwörter auswählen (siehe Abschn. 4.3.1). 


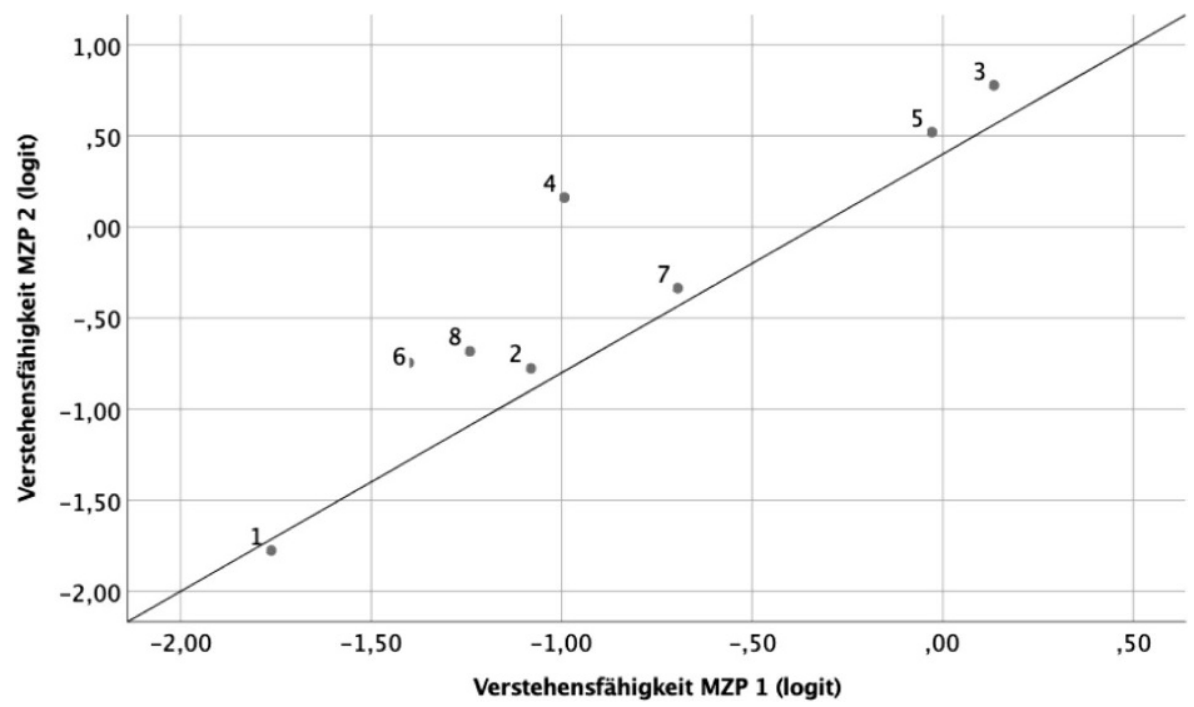

Abb. 11 Streudiagramm der Klassenmittelwerte zur Fähigkeit mathematische Textaufgaben zu verstehen im ersten und zweiten Messzeitpunkt

\subsection{Einflussfaktoren sprachbasierter Leistung(sveränderung)en (Forschungsfrage III)}

\subsubsection{Einflussfaktoren auf das Textaufgabenverständnis am Ende der Förderung}

Zur Klärung der Frage (FF III), inwieweit die Fähigkeit, Textaufgaben zu verstehen, am Ende der Förderung mit der Einschätzung unterrichtlicher Sprachförderaspekte (siehe FF I) und spezifischer personenbezogener Merkmale zusammenhängt, wurden verschiedene Regressionsmodelle gerechnet. Die Analysen belegen, dass sich für die fünf unterrichtlichen Aspekte aus FF1 sprachliche Verständlichkeit, Bedeutung von Sprache, Umgang mit sprachlichen Fehlern, Einsatz von Textverstehensstrategien und Haltung gegenüber fachlichen Sprachhandlungen sowie für die verschiedenen Hintergrundmerkmale auf Personenebene (Lesekompetenz, mathematische Fachleistung, Geschlecht, Muttersprache, gesprochene Sprache zuhause, Jahrgangsstufe, Mathematik- sowie Deutschnote) keine Zusammenhänge zum Textaufgabenverständnis am Ende der Förderung zeigen. Das Regressionsmodell in Tab. 7, bei dem wie bei allen hier präsentierten Regressionsmodellen die Prädiktoren nach Einfluss inkludiert wurden, zeigt mit der mathematischen Textverstehenskompetenz zum zweiten Messzeitpunkt als abhängige Variable, dass die einzigen Variablen mit einem signifikanten Betakoeffizienten das diesbezügliche Vorwissen zum Messzeitpunkt $1(\beta=0,424)$ sowie die über den C-Test gemessene Sprachfähigkeit zum Messzeitpunkt $1(\beta=268)$ sind. Insgesamt kann mit dem Modell dieser Regressionsanalysen $40 \%$ Varianz des Textaufgabenverständnisses zum zweiten Messzeitpunkt erklärt werden. 


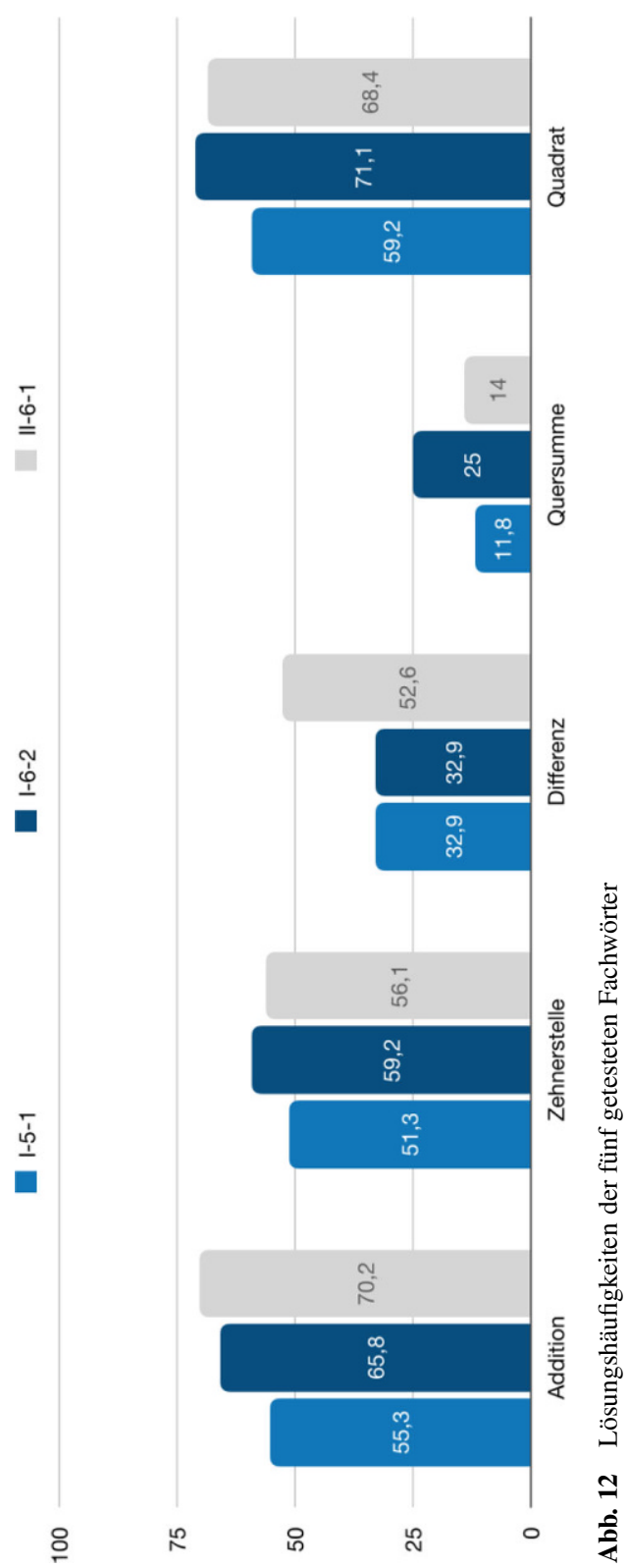

\subsubsection{Zusammenhänge zwischen Fachwortschatz, personenbezogenen} Fähigkeitsmaßen und Einstellungen

Analysiert man zunächst, welche Einflussfaktoren mit dem Vorhandensein eines hohen mathematischen Fachwortschatzes zum ersten Messzeitpunkt zusammenhängen, so zeigen zunächst bivariate Korrelationen multiple Zusammenhänge ausschließlich 


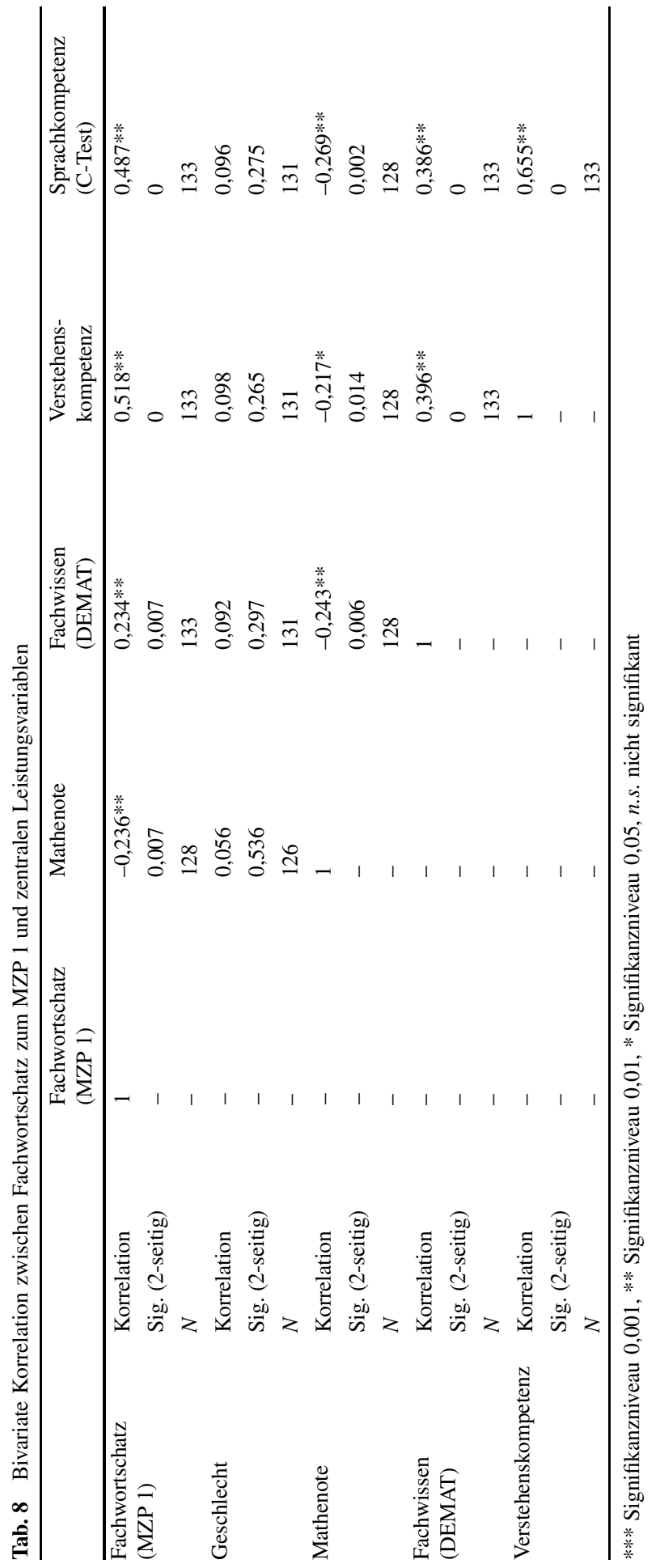


Tab. 9 Regressionsanalysen zum Fachwortschatz MZP 2 und (signifikanten) Kovariaten

\begin{tabular}{|c|c|c|c|c|c|c|c|c|}
\hline \multirow[b]{2}{*}{$\begin{array}{l}\text { Abhängige Variable: } \\
\text { Fachwortschatz (2. MZP) }\end{array}$} & \multicolumn{4}{|c|}{ Modell 1} & \multicolumn{4}{|c|}{ Modell 2} \\
\hline & $\beta$ & $\begin{array}{l}\text { Stand. } \\
\beta\end{array}$ & SE & Sig & $\beta$ & $\begin{array}{l}\text { Stand. } \\
\beta\end{array}$ & SE & Sig \\
\hline (Konstante) & 0,271 & - & 0,152 & 0,079 & 1,245 & - & 0,845 & 0,148 \\
\hline Fachwortschatz (1. MZP) & 0,594 & $0,509 * * *$ & 0,117 & 0,000 & 0,464 & $0,458 * *$ & 0,126 & 0,001 \\
\hline Geschlecht (MZP 1) & - & - & - & - & 0,703 & $0,261 *$ & 0,304 & 0,025 \\
\hline $\begin{array}{l}\text { Einstellung gegenüber } \\
\text { Sprachhandlungen im } \\
\text { Mathematikunterricht } \\
\text { (MZP 1) }\end{array}$ & - & - & - & - & $-0,320$ & $-0,249$ & 0,164 & 0,057 \\
\hline Mathematiknote (MZP 1) & - & - & - & - & $-0,192$ & $-0,220$ & 0,102 & 0,065 \\
\hline - & \multicolumn{4}{|c|}{$N=76 ; \mathrm{R}^{2}=0,26$} & \multicolumn{4}{|c|}{$N=76 ; \mathrm{R}^{2}=0,46$} \\
\hline
\end{tabular}

*** Signifikanzniveau 0,001, ** Signifikanzniveau 0,01, * Signifikanzniveau 0,05, n.s. nicht signifikant

mit Leistungsindikatoren (siehe Tab. 8) - allerdings keinerlei Zusammenhang mit der sprachbezogenen Wahrnehmung des Mathematikunterrichts oder spezifischen Personenmerkmalen wie z. B. Geschlecht, Muttersprache oder SES.

Bei der Betrachtung, inwiefern welche Variablen mit einem hohen Fachwortschatz zum zweiten Messzeitpunkt einhergehen, gilt es zu berücksichtigen, dass lediglich Daten für MZP 1 \& 2 derjenigen Probanden vorliegen, die zu Beginn des Projekts in der 5. Jahrgangsstufe waren. Entsprechend basieren die folgenden Regressionsanalysen lediglich auf diesen 76 Schülerinnen und Schülern. Dabei zeigen die Berechnungen, dass erwartungsgemäß das Vorwissen einen maßgeblichen Einfluss hat, aber lediglich $26 \%$ der Varianz alleine aufklärt (siehe Tab. 9 Modell 1). Ein entsprechend erweitertes Modell, das zwar deutlich mehr Varianz aufklärt, aber dessen Signifikanzniveaus nicht durchgängig zufriedenstellend sind, verdeutlicht, dass anscheinend die Jungen bzw. die Leistungsstärkeren Zuwächse erzielen und insbesondere diejenigen, die zu Beginn der Sprachförderung mathematischen Sprachhandlungen skeptisch gegenüberstanden, am Ende einen höheren Fachwortschatz aufweisen (siehe Tab. 9 - Modell 2).

Keine statistisch bedeutsame Rolle spielen in diesen Modellen u.a. die Faktoren: Sprachkompetenz (C-Test), Fachwissen (DEMAT), SES, Migrationshintergrund, Muttersprache sowie die Einschätzung bezüglich der sprachlichen Verständlichkeit des Mathematikunterrichts, der unterrichtlichen Bedeutsamkeit von Sprache, dem unterrichtlichen Umgang mit Fehlern und der Verwendung von Strategien auf Schülerseite.

\section{Diskussion}

In der dargestellten Evaluationsstudie wurde untersucht, inwiefern ein am BiSSForschungsvorhaben teilnehmender Verbund bestehend aus acht Klassen von einer über ein Jahr laufenden Sprachförderung profitiert. Dadurch sollte 
- ein bestehendes Forschungsdesiderat im Bereich der evidenzbasierten Sprachförderung im Fach angegangen werden (Wissenschaft),

- stellvertretend für die verschiedenen Maßnahmen in den Bundesländern exemplarisch aufgezeigt werden, inwiefern aktuelle Fördermaßnahmen auch entsprechend messbare Effekte erzielen und damit ihre durchaus ressourcenintensiven Implementationen legitimieren (Bildungspolitik)

- und letztlich galt es, zumindest Impulse - wenn auch keine empirisch überprüften Handlungsanweisungen - für die Optimierung bzw. Gestaltung praktischer Sprachfördermaßnahmen im Fach abzuleiten (schulische Praxis).

Hierfür wurden ergänzend zu qualitativ-prozessbegleitenden Verfahren, über die an anderer Stelle berichtet wird, im Rahmen einer quantitativen Erhebung im PrePost-Design verschiedene Tests und Befragungen zu Beginn und am Ende der ungefähr einjährigen Sprachförderphase im Fach Mathematik durchgeführt. Neben Standardverfahren zur Erfassung der mathematischen Leistungsstärke, der sprachliche Fähigkeiten und der sprachbezogenen Einschätzungen des Unterrichts wurden - aufgrund der verbundspezifischen Ausrichtung der Sprachförderung - der Fachwortschatz sowie sprachbezogene Verstehensleistungen als zentrale sprachbezogene Leistungskonstrukte analysiert.

Zunächst zeigen jedoch die Ergebnisse der Befragungen, dass Schülerinnen und Schüler ihren Unterricht differenziert wahrnehmen und für Veränderungen durchaus sensibel sind, wobei bei der Interpretation dieser Daten bedacht werden muss, dass zu MZP 1 die Sprachförderung bereits begonnen hatte und gerade die eher im Mittelfeld zu verortenden Skalenwerte möglicherweise vor der Sprachförderung sich auf einem anderen Niveau befanden. Dies berücksichtigend kann zunächst deskriptiv festgehalten werden, dass zwar bezüglich vier der fünf eingesetzten Skalen zur Unterrichtswahrnehmung keine Veränderungen zwischen den beiden Messzeitpunkten festgestellt werden können, allerdings geht der vermehrte Einsatz von angeleiteten Sprachhandlungen im Mathematikunterricht, als einem der beiden Sprachförderschwerpunkte des Verbundes, damit einher, dass die Schülerinnen und Schüler diesen signifikant weniger positiv gegenüber stehen als noch in der ersten Phase der Förderung. Gerade vor dem Hintergrund der Diskussion um Motivation und Volition als Teil mathematischer (Kommunikations-)Kompetenzen (vgl. Weinert 2001) wäre hier in Folgeuntersuchungen zu analysieren, inwiefern dies mit der qualitativen und quantitativen Umsetzung derartiger Sprachhandlungen im Förderunterricht zusammenhängt.

Dass die beiden Skalenwerte zur wahrgenommenen Verständlichkeit und zum Umgang mit Fehlern nicht signifikant zugenommen haben, mag an der positiv zu wertenden bereits hohen Zustimmung zu Beginn der Sprachförderung liegen. Anders verhält es sich bei der im Rahmen von fachbezogener Sprachförderung als zentral angesehenen Strategieförderung (siehe Abschn. 1.3.2). Hier sind nicht nur keine Zuwächse im Verlauf der Förderung festzustellen, sondern die Schülerinnen und Schüler machen bei der Bearbeitung der Fragebögen auch deutlich, dass für sie ein verstärkter Strategieeinsatz denkbar wäre.

Besonders bedenkenswert bei den Fragebogenanalysen erscheinen zudem die Haltungen der Schülerinnen und Schüler gegenüber mathematischen Textaufgaben. 
Nicht nur, dass zu Beginn der Sprachförderung Schülerinnen und Schülern derartigen Aufgaben eher ablehnend gegenüberstehen, auch nach einem Jahr der intensiven Auseinandersetzung gibt es hier keine signifikanten Zuwächse. Gerade die in den beiden Klassen Nr. 3 und Nr. 4 sogar deutlich gesteigerten Ressentiments belegen, dass neben den Sprachhandlungen auch in diesem Bereich der vermehrte Einsatz von vermeintlich sprachförderlichen Textaufgaben teilweise zu negativen Einstellungsveränderungen bei den Schülerinnen und Schülern geführt hat, obwohl gerade diese Klassen aufgrund der sprachlichen und mathematischen Fähigkeiten als so leistungsstark angesehen werden können, dass die empirisch eher schwierigeren Textaufgaben auf der Performanzebene keine größeren Probleme verursachen sollten. Dies erscheint nicht nur für einen sprachsensiblen Fachunterricht, sondern selbst im Rahmen eines ausschließlich mathematisch-inhaltlich bezogenen Fachunterrichts als unbefriedigende Kompetenzentwicklung.

So ambivalent wie die Ergebnisse der Befragungen erscheinen, so deutlich zeigen sich im Bereich der Leistungsentwicklung, dass die Schülerinnen und Schüler nicht nur bei spezifischen fachmathematischen Aufgabenstellungen (siehe Abschn. 4.2) im Verlauf des Schuljahres signifikante Zuwächse erzielen, sondern auch durchgängig höhere Leistungen beim Verstehen von Textaufgaben sowie bei der Verwendung des mathematischen Fachwortschatzes. Dass dabei die leistungsschwächste Klasse beim Textverständnis keinerlei Zuwächse verzeichnen kann, mag als Einzelfall gelten. Hingegen offenbaren die absoluten Lösungsraten bei den Fachwörtern am zweiten Messzeitpunkt, dass auch die explizit darauf abzielende Sprachförderung die normativen Ziele der Lehrpläne nicht zu erfüllen vermag. Hierbei gilt es zu beachten, dass diese Zuwächse beim Textverständnis bzw. dem Wortschatz neben dem diesbezüglichen Vorwissen insbesondere bei sprachstärkeren Schülerinnen und Schülern bzw. bei Jungen und fachlich Leistungsstärkeren zu verzeichnen sind.

Dass insbesondere die sprachlichen Fähigkeiten, aber nicht die sprachlich-biographischen Personenmerkmale, wie Muttersprache oder gesprochene Sprache zuhause, einen Einfluss auf das Textaufgabenverständnis zum Ende der Förderung haben, zeigt, dass keine spezifische Sprachförderung, im Sinne von Mathematikförderung für DaZ-Schülerinnen und -Schüler, sondern vielmehr eine Förderung für alle, insbesondere aber für die sprachlich schwächeren Schülerinnen und Schüler stattfinden sollte. Ergänzend hierzu zeigen auch Prediger und Wessel (2018), dass sprachlich schwache ein- und mehrsprachige Schülerinnen und Schüler dieselben Schwierigkeiten haben und somit dieselbe fachspezifische Sprachförderung benötigen.

Für die praktische Gestaltung von Sprachfördermaßnahmen lassen sich aus den hier vorgestellten Ergebnissen zumindest folgende tentative Gestaltungsprinzipien ableiten:

1. Neben eher kognitiven Elementen der fachspezifischen Sprachförderung sollte insbesondere in Unterrichtsfächern, die bisher vermeintlich eine eher geringe Nähe zu Texten bzw. Sprache aufwiesen, darauf Wert gelegt werden, dass Schülerinnen und Schüler gegenüber diesen Lernelementen eine grundsätzlich positive(re) Haltung einnehmen; gerade wenn hiermit Veränderungen in den Einstellungen bzw. beliefs verbunden sind. 
2. Insbesondere der vermehrte Einsatz neuer unterrichtlicher Elemente wie z. B. angeleitete Sprachhandlungen oder umfangreichere Textaufgaben sollte stets mit einer reflexiven Grundhaltung in den bisherigen Normalunterricht integriert werden, um nicht als künstliche oder gar störende Fremdkörper aufgefasst und entsprechend von den Schülerinnen und Schülern abgelehnt zu werden.

3. Im Rahmen der stärkeren Auseinandersetzung mit (Fach-)Sprache und eines damit evtl. einhergehenden gesteigerten sprachlichen Anspruchs muss in besonderem Maße auf die Adaptivität der Fördermaßnamen geachtet werden, damit der zweite Aspekt des sogenannten Matthäuseffekts vermieden wird ${ }^{9}$ und auch sprachlich schwächere Schülerinnen und Schüler profitieren.

4. Auch wenn Sprachfördermaßnahmen anscheinend einen umfangreicheren Einsatz von strategischen Handlungsweisen bewirken, so besteht in dieser Allgemeinheit kein - zumindest messbarer - Zusammenhang mit evtl. Leistungsveränderungen. Entsprechend vorsichtig sollten Fördermaßnahmen bei der Vermittlung von nur bedingt evidenzbasierten Förderkatalogen (z. B. Leisen 2010) sein und evtl. eher auf die wenigen bisher empirisch abgesicherten fachspezifischen Verstehensstrategien (Leiss et al. 2019; Prediger und Krögeloh 2015) fokussieren.

5. Um eine Steigerung der Effekte von Sprachfördermaßnahmen bezüglich der Einstellungen der Lehrpersonen bzw. der Schülerinnen und Schüler oder zentraler Leistungsvariabelen zu erzielen, evtl. auch nur in einem fokussierten Bereich, erscheint unter Gewährung entsprechender Ressourcen eine stärkere Integration der Lehrpersonen in die Erstellung der Sprachfördermaterialien zweckdienlich.

Scheinen die Analysen der vorliegenden Studie diese Interpretationen auch nahezulegen, so bedarf es diesbezüglich spezifischer und inhaltlich stärker fokussierter Studien, die im Rahmen eines methodisch kontrollierten Settings einzelne Aspekte untersuchen. Damit muss deutlich auf die Grenzen des vorliegenden Ansatzes hingewiesen werden. Da es sich bei der vorliegenden Studie um wissenschaftliche Begleitforschung handelt, die ein bereits geplantes Fördervorhabens evaluieren sollte, bestand nur in eingeschränktem Maße die Möglichkeit, ein klares Forschungsdesign mit speziell darauf abgestimmten Untersuchungsinstrumenten umzusetzen. Vielmehr gibt es viele Elemente, die den Anforderungen der schulischen (Förder-)Praxis genügen mussten und entsprechend wissenschaftlich nicht immer die Anforderungen an starke Designs erfüllen (Rost 2013). Als stärkstes Defizit mag hier insbesondere das Fehlen einer Kontrollgruppe erscheinen, da mit den vorliegenden Daten offen bleibt, inwiefern die gemessenen Effekte insbesondere auf das BiSS-Förderprogramm und nicht primär auf andere Variablen zurückzuführen sind. Aber auch das mangelnde Wissen, inwiefern die intendierte Sprachförderung in den einzelnen Klassen auch umgesetzt wurde, zeigt den Bedarf an ergänzenden Unterrichtsbeobachtungen und Lehrkräftebefragungen, welche aufgrund des Projektumfangs nur bedingt realisiert werden konnten. Hier gilt es eine Balance zwischen strengen wissenschaftlichen Gütekriterien und einem praxisrelevanten Erkenntnisgewinn zu wahren. Neben dieser Grundsatzproblematik gibt es allerdings weitere, insbesondere die Instrumente be-

9 „Denn wer da hat, dem wird gegeben, dass er die Fülle habe; wer aber nicht hat, dem wird auch das genommen, was er hat.“ Matthäus 25, 29 (Luther Übersetzung). 
treffende Aspekte, die auch bei einer nochmaligen Durchführung in einem ähnlichen Setting überarbeitet werden müssten. So sollte u.a.

- um das Problem der teilweise zu niedrigen Fallzahlen (z.B. durch die extreme drop-out-Rate) bei den Analysen zu verringern, ein Testdesign verwendet werden, dass über alle Jahrgänge hinweg Ankeritems verwendet,

- die Erfassung der eher negativen Einstellungen Textaufgaben gegenüber durch eine reliable Skala und nicht nur durch ein einzelnes Item erfolgen und

- das Konstrukt des mathematikaufgabenbezogenen Textverständnisses noch durch weitere inhaltlich differenziertere Operationalisierungen sowie der Fachwortschatz durch ein umfangreicheres Instrument erfasst werden.

Aspekte wie z.B. die zeitliche Verschiebung der Erhebungen im Schulhalbjahr zwischen den Klassen oder eine Kontrolle des realisierten Curriculums müsste bei zukünftigen Evaluationsprojekten unbedingt stärker als bisher in Betracht gezogen werden, auch wenn dadurch ggfs. die Anzahl der beforschten Förderverbünde einzuschränken wäre.

Bilanzierend kann festgestellt werden, dass eine derartige prozessbegleitende Evaluation eines größeren Sprachfördervorhabens wie dem BiSS-Projekt zwar wissenschaftlich mit zahlreichen Herausforderungen verbunden ist und insbesondere im methodischen Bereich der Feldforschung mit inhaltlich und organisatorisch unterschiedlichen, aber parallel ablaufenden Interventionsstudien noch viele offen Fragen bestehen, aber dessen ungeachtet sowohl gegenüber der Bildungspolitik eine gewisse Rechtfertigung derartiger Vorhaben darstellt als auch der schulischen Praxis relevante Impulse oder Warnungen liefern kann.

Funding Open Access funding provided by Projekt DEAL.

Open Access Dieser Artikel wird unter der Creative Commons Namensnennung 4.0 International Lizenz veröffentlicht, welche die Nutzung, Vervielfältigung, Bearbeitung, Verbreitung und Wiedergabe in jeglichem Medium und Format erlaubt, sofern Sie den/die ursprünglichen Autor(en) und die Quelle ordnungsgemäß nennen, einen Link zur Creative Commons Lizenz beifügen und angeben, ob Änderungen vorgenommen wurden.

Die in diesem Artikel enthaltenen Bilder und sonstiges Drittmaterial unterliegen ebenfalls der genannten Creative Commons Lizenz, sofern sich aus der Abbildungslegende nichts anderes ergibt. Sofern das betreffende Material nicht unter der genannten Creative Commons Lizenz steht und die betreffende Handlung nicht nach gesetzlichen Vorschriften erlaubt ist, ist für die oben aufgeführten Weiterverwendungen des Materials die Einwilligung des jeweiligen Rechteinhabers einzuholen.

Weitere Details zur Lizenz entnehmen Sie bitte der Lizenzinformation auf http://creativecommons.org/ licenses/by/4.0/deed.de. 


\section{Anhang}

\section{Skalen der Schüler*innen-Fragebögen}

\section{Items der Skala Sprachliche Verständlichkeit}

4-Punkt-Likert-Skala $(1=$ stimmt gar nicht, $2=$ stimmt eher nicht, $3=$ stimmt eher, 4 = stimmt ganz genau)

I. „Die Aufgabenstellungen im Mathematikunterricht sind für mich klar und verständlich.“

II. „Wenn mein Mathematiklehrer/meine Mathematiklehrerin etwas erklärt, dann gibt er/sie dazu anschauliche Beispiele."

III. „Mein Mathematiklehrer/meine Mathematiklehrerin drückt sich klar und deutlich aus.“

Items der Skala Allgemeine Bedeutung von Sprache

4-Punkt-Likert-Skala $\quad(1=$ stimmt gar nicht, $2=$ stimmt eher nicht, $3=$ stimmt eher, $4=$ stimmt ganz genau)

I. „Mein Mathematiklehrer/meine Mathematiklehrerin weist darauf hin, wie nützlich gutes Deutsch im Alltag ist.“

II. „Mein Mathematiklehrer/meine Mathematiklehrerin betont, dass gutes Deutsch in vielen Berufen eine große Rolle spielt.“

III. „Mein Mathematiklehrer/meine Mathematiklehrerin betont, dass heutzutage jeder in der Lage sein muss, sich im Deutschen gut auszudrücken.“

Items der Skala Umgang mit Sprachlichen Fehlern

4-Punkt-Likert-Skala $\quad(1=$ stimmt gar nicht, $2=$ stimmt eher nicht, $3=$ stimmt eher, 4 = stimmt ganz genau)

I. „Wenn ich im Unterricht sprachliche Fehler mache, besprechen meine Lehrerinnen und Lehrer diese mit mir auf eine Art und Weise, dass es mir wirklich etwas bringt."

II. „Wenn ich ein falsches Wort benutze, erklären meine Lehrerinnen und Lehrer, was daran falsch ist."

III. „Meine Lehrerinnen und Lehrer sind überzeugt, dass sprachliche Fehler nützlich sind, weil wir dararaus lernen können.“

IV. „Wenn ich mit der Sprache etwas falsch mache, erhalte ich die Möglichkeit, mich zu korrigieren oder noch einmal anzufangen."

V. „Aus der Verbesserung meiner Sprache im Unterricht durch meine Lehrerin/ meinen Lehrer lerne ich etwas dazu.“ 
Items der Skala Einsatz von Textverstehensstrategien

4-Punkt-Likert-Skala $\quad(1=$ nie, $2=$ selten, $3=$ oft, $4=$ fast immer $)$

„Wie gehst du vor, wenn du Textaufgaben bearbeitest, bei denen du den Lösungsweg nicht gleich erkennst?

Normalerweise, wenn ich solche Textaufgaben bearbeite ...“

I. , ,... halte ich fest, welche Informationen gegeben und gesucht sind.“

II. „,... überlege ich, welche Informationen im Text wichtig und unwichtig sind.“

III. „,... versuche ich, mir die Situation, die im Text beschrieben ist, möglichst genau vorzustellen“

IV. ,,... versuche ich, herauszufinden, wie die Angaben in der Aufgabe mit miteinander zusammenhängen.“

V. ,... lese ich den Text mehrfach durch.“

Items der Skala Haltung gegenüber mathematischen Sprachhandlungen

5-Punkt-Likert-Skala ( $1=$ stimmt überhaupt nicht, $2=$ stimmt eher nicht, $3=$ stimmt teils, teils, $4=$ stimmt überwiegend, $5=$ stimmt genau)

I. Ich spreche mit Mitschülern über meine eigenen Lösungswege.“

II. „Ich schreibe meinen Lösungswege gern auf.“

III. ,Ich höre gerne zu, wenn Mitschüler ihre Lösungswege vorstellen.“

\section{Items zur Messung des Sprachwortschatzes}

Items Fachwortschatz „Addition“

\section{die Addition}

Was bedeutet der Begriff Addition?

Kreuze die einzig richtige Antwort an.

$\square$ Der Begriff Addition bedeutet, dass Zahlen voneinander abgezogen werden.

$\square$ Der Begriff Addition bedeutet, dass eine Zahl mit der Zahl zwei vervielfacht wird.

$\square$ Der Begriff Addition bedeutet, dass Zahlen zusammengezählt werden.

$\square$ Der Begriff Addition bezeichnet die Zahl, die entsteht, wenn man Zahlen zusammenzählt.

$\square$ Der Begriff Addition bezeichnet die Zahl, die entsteht, wenn man Zahlen voneinander abzieht. 


\section{Item „Zehnerstelle“}

\section{die Zehnerstelle}

Unterstreiche bei der nachfolgenden Zahl die Zehnerstelle.

Unterstreiche die einzig richtige Antwort.

\section{7}

\section{Items Fachwortschatz „Differenz“}

\section{Welcher Begriff wird gesucht?}

Wenn du eine Zahl von einer anderen Zahl abziehst erhältst du...

Kreuze die einzig richtige Antwort an.
$\square$ ein Produkt.
$\square$ eine Summe.
$\square$ einen Quotienten.
$\square$ einen Divisor.
$\square$ eine Differenz.

Items Fachwortschatz „Quersumme“

\section{die Quersumme}

Was ist die Quersumme?

Kreuze die einzig richtige Antwort an.

$\square$ Die Quersumme einer Zahl erhalte ich, wenn ich die geraden Ziffern einer Zahl addiere.

$\square$ Die Quersumme einer Zahl erhalte ich, wenn ich alle Ziffern einer Zahl multipliziere.

$\square$ Die Quersumme einer Zahl erhalte ich, wenn ich alle Ziffern einer Zahl addiere.

$\square$ Die Quersumme einer Zahl erhalte ich, wenn ich die ungeraden Ziffern einer Zahl addiere.

$\square$ Die Quersumme einer Zahl erhalte ich, wenn ich alle Ziffern einer Zahl durch die Zahl 2 teile. 


\section{Items Fachwortschatz „Quadrat“}

\begin{tabular}{|c|} 
das Quadrat \\
\hline Was ist ein Quadrat? \\
\hline
\end{tabular}

\section{Kreuze die einzig richtige Antwort an.}

\begin{tabular}{|l|l|}
\hline$\square$ & \\
\hline$\square$ & \\
\hline & \\
\hline & \\
\hline & \\
\hline & \\
\hline & \\
\hline
\end{tabular}

\section{Literatur}

Abedi, J. (2006). Language issues in item development. In S. M. Downing \& T. M. Haladyna (Hrsg.), Handbook of test development (S. 377-398). Mahwah: Erlbaum.

Abedi, J., \& Leon, S. (1999). Impact of students' language background variables on content-based performance: analyses and extent data

Abedi, J., Leon, S., Wolf, M. K., \& Farnsworth, T. (2008). Detecting test items differentially impacting the performance of ELL students. In M. K. Wolf, J.L. Herman, J. Kim, J. Abdi, S. Leon \& N. Griffin, et al. (Hrsg.), Providing validity evidence to improve the assessment of english language learners (S. 55-80).

Ahrenholz, B. (2017). Sprache in der Wissensvermittlung und Wissensaneignung im schulischen Fachunterricht. Empirische Einblicke. In B. Lütke, I. Petersen \& T. Tajmel (Hrsg.), Fachintegrierte Sprachbildung: Forschung, Theoriebildung und Konzepte für die Unterrichtspraxis (S. 1-32). Berlin: de Gruyter.

Becker-Mrotzek, M., Schramm, K., Thürmann, E., \& Vollmer, H. J. (2013). Sprache im Fach: Sprachlichkeit und fachliches Lernen. Fachdidaktische Forschungen, Bd. 3. Münster: Waxmann.

Becker-Mrotzek, M., Hentschel, B., Hippmann, K., \& Linnemann, M. (2012). Sprachförderung in deutschen Schulen - Die Sicht der Lehrerinnen und Lehrer. Eine Umfrage unter Lehrerinnen und Lehrern. http://www.mercator-institut-sprachfoerderung.de/fileadmin/user_upload/Lehrerumfrage_ Langfassung_final_30_05_03.pdf. Zugegriffen: 8. Januar 2020 
Blum, W., \& Leiss, D. (2005). Modellieren im Unterricht mit der „Tanken“-Aufgabe. Mathematik Lehren, $128,18-21$.

Bodenburg, T., Key, C., \& Krüll, L. (2018). Anteile der Schüler mit Zuwanderungsgeschichte in NRW gestiegen. https://www.it.nrw/node/91716/pdf. Zugegriffen: 19. Juni 2019.

Bos, W., Bonsen, M., Kummer, N., Lintorf, K., \& Frey, K. (2009). TIMSS 2007. Dokumentation der Erhebungsinstrumente zur Trends in International Mathematics and Science Study. Münster: Waxmann.

Bos, W., Lankes, E. M., Prenzel, M., Schwippert, K., Walther, G., \& Valtin, R. (2003). Erste Ergebnisse aus IGLU. Schülerleistungen am Ende der vierten Jahrgangsstufe im internationalen Vergleich. Münster: Waxmann.

Bos, W., Strietholt, R., Goy, M., Stubbe, T., Tarelli, I., \& Hornberg, S. (2010). IGLU 2006. Dokumentation der Erhebungsinstrumente. Münster: Waxmann.

Bühner, M., \& Ziegler, M. (2017). Statistik für Psychologen und Sozialwissenschaftler. München: Pearson Studium.

Clarkson, P.C. (1991). Language comprehension errors: a further investigation. Mathematics Education Research Journal, 3(2), 24-33.

Deutsches Institut für Internationale Pädagogische Forschung (2009). Deutsch Englisch Schülerleistungen International. Schülerfragebogen 3. Frage 6 und Frage 10. https://daqs.fachportal-paedagogik. de/download/index/file_id/35. Zugegriffen: 12. Mai 2016.

Duarte, J., Gogolin, I., \& Kaiser, G. (2011). Sprachlich bedingte Schwierigkeiten von mehrsprachigen Schülerinnen und Schülern bei Textaufgaben. In E. Özdil \& S. Prediger (Hrsg.), Mathematiklernen unter Bedingungen der Mehrsprachigkeit. Stand und Perspektive der Forschung und Entwicklung in Deutschland (S. 35-54). Münster: Waxmann.

Galbraith, P., \& Stillman, G. (2006). A framework for identifying student blockages during transitions in the modelling process. ZDM-International Journal on Mathematics Education, 38(2), 143-162. https://doi.org/10.1007/BF02655886.

Gebhardt, M., Rauch, D., Mang, J., Sälzer, C., \& Stanat, P. (2013). Mathematische Kompetenz von Schülerinnen und Schülern mit Migrationshintergrund. In M. Prenzel, C. Sälzer, E. Klieme \& O. Köller (Hrsg.), PISA 2012. Fortschritte und Herausforderungen in Deutschland (S. 275-308). Münster, New York: Waxmann.

Gogolin, I., \& Lange, I. (2011). Bildungssprache und durchgängige Sprachbildung. In S. Fürstenau \& M. Gomolla (Hrsg.), Migration und schulischer Wandel. Mehrsprachigkeit (S. 107-129). Wiesbaden: VS.

Grotjahn, R. (2014). Der C-Test: Aktuelle Tendenzen. Frankfurt a.M.: Peter Lang.

Haag, N., Heppt, B., Stanat, P., Kuhl, P., \& Pant, H. A. (2013). Second language learners' performance in mathematics: disentangling the effects of academic language features. Learning and Instruction, 28, 24-34. https://doi.org/10.1016/j.learninstruc.2013.04.001.

Hagena, M., Leiss, D., \& Schwippert, K. (2017). Using reading strategy training to foster students' mathematical modelling competencies: Results of a quasi-experimental control trial. Eurasia Journal of Mathematics, Science and Technology Education, 13(7), 4057-4085. https://doi.org/10.12973/ eurasia.2017.00803a.

Harsch, C., \& Hartig, J. (2010). Empirische und inhaltliche Analyse lokaler Abhängigkeiten im C-Test. In R. Grotjahn (Hrsg.), Der C-Test: Beiträge aus der aktuellen Forschung (S. 193-204). Frankfurt a.M.: Peter Lang.

Heinze, A., Herwartz-Emden, L., Braun, C., \& Reiss, K. (2011). Die Rolle von Kenntnissen der Unterrichtssprache beim Mathematiklernen: Ergebnisse einer quantitativen Längsschnittstudie in der Grundschule. In S. Prediger \& E. Özdil (Hrsg.), Mathematiklernen unter Bedingungen der Mehrsprachigkeit. Stand und Perspektiven der Forschung und Entwicklung in Deutschland. Mehrsprachigkeit, (Bd. 32, S. 11-33). Münster: Waxmann.

Heinze, A., Herwartz-Emden, L., \& Reiss, K. (2007). Mathematikkenntnisse und sprachliche Kompetenz bei Kindern mit Migrationshintergrund zu Beginn der Grundschulzeit. Zeitschrift Für Pädagogik, 53(4), 562-581.

Henschel, S., Gentrup, S., Beck, L., \& Stanat, P. (Hrsg.). (2018). Projektatlas Evaluation: Erste Ergebnisse aus den BiSS-Evaluationsprojekten. Berlin: BiSS-Trägerkonsortium.

Klieme, E., Pauli, C., \& Reusser, K. (2005). Dokumentation der Erhebungs- und Auswertungsinstrumente zur schweizerisch-deutschen Videostu-die. „Unterrichtsqualität, Lernverhalten und mathematisches Verständnis “. 1. Befragungsinstrumente. Frankfurt a.M.: GEBF.

Koch-Priewe, B., \& Krüger-Potratz, M. (2016). Qualifizierung für sprachliche Bildung. Die Deutsche Schule, 13, 9-20. 
Leisen, J. (2010). Handbuch Sprachförderung. Sensibler Fachunterricht in der Praxis. Grundlagenteil. Stuttgart: Klett.

Leiss, D., \& Blum, W. (2006). Beschreibung zentraler mathematischer Kompetenzen. In W. Blum (Hrsg.), Bildungsstandards Mathematik: konrekt, Sekundarstufe I: Aufgabenbeispiele, Unterrichtsanregungen, Fortbildungsideen (S. 33-50). Berlin: Cornelsen.

Leiss, D., Domenech, M., Ehmke, T., \& Schwippert, K. (2017). Schwer - schwierig - diffizil: Zum Einfluss sprachlicher Komplexität von Aufgaben auf fachliche Leistungen in der Sekundarstufe 1. In D. Leiss, M. Hagena, A. Neumann \& K. Schwippert (Hrsg.), Sprache im Fach Mathematik_Forschungsstand und Herausforderungen im Verlauf der Schulzeit (S. 99-126). Münster: Waxmann.

Leiss, D., Hagena, M., Neumann, A., \& Schwippert, K. (2015). Sprache im Mathematikunterricht. Eine Bestandsaufnahme des aktuellen didaktischen Diskurses. In D. Leiss, M. Hagena, A. Neumann \& K. Schwippert (Hrsg.), Mathematik und Sprache. Empirischer Forschungsstand und unterrichtliche Herausforderungen (S. 11-42). Münster: Waxmann.

Leiss, D., Plath, J., \& Schwippert, K. (2019). Language and mathematics-Key factors influencing the comprehension process in reality-based tasks. Mathematical Thinking and Learning, 21(2), 131-153. https://doi.org/10.1080/10986065.2019.1570835.

Leiss, D., Schukajlow, S., Blum, W., Messner, R., \& Pekrun, R. (2010). Zur Rolle des Situationsmodells beim mathematischen Modellieren - Aufgabenanalysen, Schülerkompetenzen und Lehrerinterventionen. Journal Für Mathematik-Didaktik, 31(1), 119-141. https://doi.org/10.1007/s13138-010-0006y.

Limbird, C., \& Stanat, P. (2006). Sprachförderung bei Schülerinnen und Schülern mit Migrationshintergrund: Ansätze und ihre Wirksamkeit. In J. Baumert, P. Stanat \& R. Watermann (Hrsg.), Herkunftsbedingte Disparitäten im Bildungswesen: Differenzielle Bildungsprozesse und Probleme der Verteilungsgerechtigkeit: Vertiefende Analysen im Rahmen von PISA 2000 (S. 257-307). Wiesbaden: VS.

Lucas, T., \& Villegas, A. M. (2011). A framework for preparing linguistically responsive teachers. In T. Lucas (Hrsg.), Teacher preparation for linguistically diverse classrooms (S. 55-72). New York: Routledge.

Maier, H., \& Schweiger, F. (1999). Mathematik und Sprache: Zum Verstehen und Verwenden von Fachsprache im Mathematikunterricht. Wien: oebv + hpt.

Martiniello, M. (2009). Linguistic complexity, schematic representations, and differential item functioning for English language learners in math tests. Educational Assessment, 14(3), 160-179. https://doi.org/ 10.1080/10627190903422906.

Mayer, R.E., \& Hegarty, M. (1996). The process of understanding mathematical problems. In R. J. Sternberg \& T. Ben-Zeev (Hrsg.), Studies in mathematical thinking and learning series. The nature of mathematical thinking (S. 29-53). Mahwah: Erlbaum.

Michalak, M. (2014). Sprache als Lernmedium im Fachunterricht. Theorien und Modelle für das sprachbewusste Lehren und Lernen. Baltmannsweiler: Schneider Hohengehren.

Michalak, M., Lemke, V., \& Goeke, M. (2015). Sprache im Fachunterricht. Eine Einführung in Deutsch als Zweitsprache und sprachbewussten Unterricht. Tübingen: Narr Francke Attempto.

Ministerium für Schule und Weiterbildung, Wissenschaft und Forschung des Landes Nordrhein-Westfalen (1999). Förderung in der deustchen Sprache als Aufgabe des Unterrichts in allen Fächern. Empfehlungen. Frechen: Ritterbach.

Ministerium für Schule, Jugend und Kinder des Landes Nordrhein-Westfalen (2004). Kernlehrplan für die Gesamtschule - Sekundarstufe I in Nordrhein-Westfalen. Frechen: Ritterbach.

Morek, M., \& Heller, V. (2012). Bildungssprache - Kommunikative, epistemische, soziale und interaktive Aspekte ihres Gebrauchs. Zentralblatt Für Didaktik Der Mathematik, 57(1), 67-101.

Mücke, S. (2007). Einfluss personeller Eingangsvoraussetzungen auf Schülerleistungen im Verlauf der Grundschulzeit. In K. Möller, P. Hanke, C. Beinbrech, R. Hein, A. K. Kleickmann \& T. Schages (Hrsg.), Qualität von Grundschulunterricht: entwickeln, erfassen und bewerten (S. 277-280). Wiesbaden: VS.

National Council of Teachers of Mathematics (2000). Principles and standards for school mathematics. Reston: Graphic Arts Center.

Naumann, J., Artelt, C., Schneider, W., \& Stanat, P. (2010). Lesekompetenz von PISA 2000 bis PISA 2009. In E. Klieme, C. Artelt, J. Hartig, N. Jude \& O. K. ller, et al. (Hrsg.), PISA 2009. Bilanz nach einem Jahrzehnt (S. 23-71). Münster: Waxmann.

Niss, M. (2003). Mathematical competencies and the learning of mathematics: the Danish KOM project. In A. Gagatsis \& S. Papastavridis (Hrsg.), 3rd Mediterranean Conference on Mathematical Education. Athens-Hellas 3-4-5 January (S. 115-124). 
Paetsch, J., \& Felbrich, A. (2016). Longitudinale Zusammenhänge zwischen sprachlichen Kompetenzen und elementaren mathematischen Modellierungskompetenzen bei Kindern mit Deutsch als Zweitsprache. Psychologie in Erziehung Und Unterricht, 63(1), 16-33. https://doi.org/10.2378/peu2016. art03d.

Paetsch, J., Radmann, S., Felbrich, A., Lehmann, R., \& Stanat, P. (2016). Sprachkompetenz als Prädiktor mathematischer Kompetenzentwicklung von Kindern deutscher und nicht-deutscher Familiensprache. Zeitschrift Für Entwicklungspsychologie Und Pädagogische Psychologie, 48(1), $27-41$.

Pimm, D. (1987). Speaking mathematically: Communication in mathematics classrooms. Language, education, and society. London: Routledge.

Plath, J., \& Leiss, D. (2018). The impact of linguistic complexity on the solution of mathematical modelling tasks. Zdm Mathematics Education, 50(1/2), 159-172. https://doi.org/10.1007/s11858-017-0897-x.

Prediger, S., \& Krögeloh, N. (2015). Low achieving eighth graders learn to crack word problems: a design research project for aligning a strategic scaffolding tool to students' mental processes. ZDM Mathematics Education, 47(6), 947-962.

Prediger, S., \& Wessel, L. (2018). Brauchen mehrsprachige Jugendliche eine andere fach- und sprachintegrierte Förderung als einsprachige? Differentielle Analysen zurWirksamkeit zweier Interventionen in Mathematik. Zeitschrift Für Erziehungswissenschaft, 21, 361-382. https://doi.org/10.1007/s11618017-0785-8.

Prediger, S., Wilhelm, N., Büchter, A., Gürsoy, E., \& Benholz, C. (2015). Sprachkompetenz und Mathematikleistung - Empirische Untersuchung sprachlich bedingter Hürden in den Zentralen Prüfungen 10. Journal Für Mathematik-Didaktik, 36(1), 77-104. https://doi.org/10.1007/s13138-015-0074-0.

Redder, A., Schwippert, K., Hasselhorn, M., Forschner, S., Fickermann, D., \& Ehlich, K. (2010). Grundzüge eines nationalen Forschungsprogramms zu Sprachdiagnostik und Sprachförderung. ZUSE-Diskussionspapier, Bd. 1. Hamburg: ZUSE.

Rösch, H., \& Paetsch, J. (2011). Sach- und Textaufgaben im Mathematikunterricht als Herausforderung für mehrsprachige Kinder. In S. Prediger \& E. Özdil (Hrsg.), Mathematiklernen unter Bedingungen der Mehrsprachigkeit. Stand und Perspektive der Forschung und Entwicklung in Deutschland (S. 55-76). Münster: Waxmann.

Rost, D. H. (2013). Interpretation und Bewertung pärdagogisch-psychologischer Studien. Eine Einführung. Bad Heilbrunn: Klinkhardt.

Schilcher, A., Röhrl, S., \& Krauss, S. (2017). Sprache im Mathematikunterricht. Eine Bestandsaufnahme des aktuellen didaktischen Diskurses. In D. Leiss, M. Hagena, A. Neumann \& K. Schwippert (Hrsg.), Mathematik und Sprache. Empirischer Forschungsstand und unterrichtliche Herausforderungen (S. 11-42). Münster: Waxmann.

Schmölzer-Eibinger, S., Dorner, M., Langer, E., \& Helten-Pacher, M. (2013). Sprachförderung im Fachunterucht in sprachlich heterogenen Klassen. Stuttgart: Klett.

Schulz-Heidorf, K. (2016). Individuelle Förderung im Unterricht: Eine Möglichkeit, soziale Herkunft und Schulerfolg zu entkoppeln? Eine Re-Analyse aus IGLU-E 2011. Berlin:: epubli.

Shaftel, J., Belton-Kocher, E., Glasnapp, D., \& Poggio, J. (2006). The impact of language characteristics in mathematics test items on the performance of English language learners and students with disabilities. Educational Assessment, 11(2), 105-126. https://doi.org/10.1207/s15326977ea1102_2.

Stanat, P., Rauch, D., \& Segeritz, M. (2010). Schülerinnen und Schüler mit Migrationshintergrund. In E. Klieme, C. Artelt, J. Hartig \& N. Jude, et al. (Hrsg.), PISA 2009. Bilanz nach einem Jahrzehnt (S. 200-228). Münster: Waxmann.

Verschaffel, L., Greer, B., \& De Corte, E. (2000). Making sense of word problems. Contexts of learning. Lisse, Exton: Swets \& Zeitlinger.

Vukovic, R. K., \& Lesaux, N. K. (2013). The language of mathematics: Investigating the ways language counts for children's mathematical development. Journal of Experimental Child Psychology, 115(2), 227-244. https://doi.org/10.1016/j.jecp.2013.02.002.

Wagner, W., Helmke, A., \& Rösner, E. (2009). Deutsch Englisch Schülerleistungen International. Dokumentation der Erhebungsinstrumente für Schülerinnen und Schüler, Eltern und Lehrkräfte. Frankfurt a.M.: GEBF, DIPF.

Walzeburg, A. (2014). Sprachlich bedingte soziale Ungleichheit: Theoretische und empirische Betrachtungen am Beispiel mathematischer Testaufgaben und ihrer Bearbeitung. Münster: Waxmann.

Weinert, F.E. (Hrsg.). (2001). Leistungsmessung in Schulen. Weinheim, Basel: Beltz.

Wendt, H., Schwippert, K., \& Stubbe, T.C. (2016). Mathematische und naturwissenschaftliche Kompetenzen von Schülerinnen und Schülern mit Migrationshintergrund. In H. Wendt, W. Bos, C. Selter, O. Köller, K. Schwippert \& D. Kasper (Hrsg.), TIMSS 2015. Mathematische und naturwis- 
senschaftliche Kompetenzen von Grundschulkindern in Deutschland im internationalen Vergleich 2 (S. 317-332). Münster, New York: Waxmann.

Wijaya, A., van den Heuvel-Panhuizen, M., Doorman, M., \& Robitzsch, A. (2014). Difficulties in solving context-based PISA mathematics tasks: An analysis of students' errors. The Mathematics Enthusiast, 11(3), 555-584.

Wolf, M.K., \& Leon, S. (2009). An investigation of the language demands in content assessments for English language learners. Educational Assessment, 14(3-4), 139-159. https://doi.org/10.1080/ 10627190903425883. 